
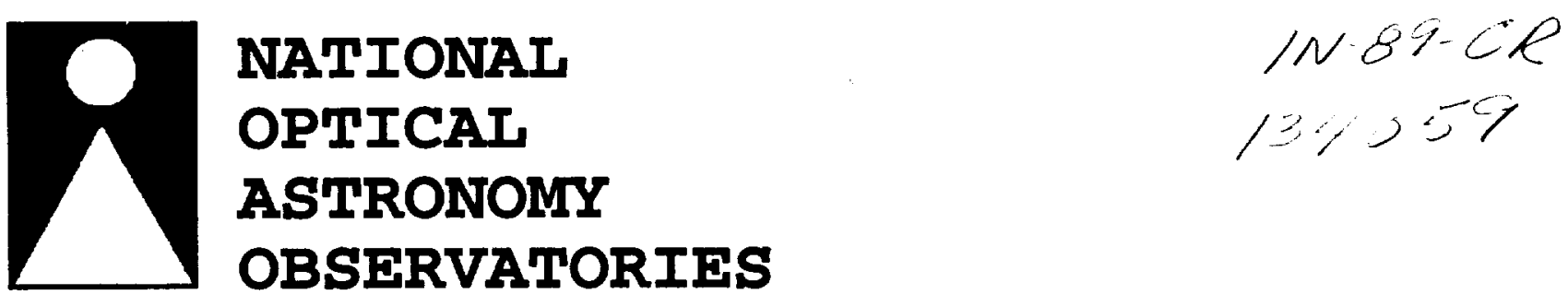

Preprint Series

NOAO Preprint No. 775

X-ray Activity in the Open Cluster IC 4665

Mark S. Giampapa

Charles F. Prosser

Thomas A. Fleming

Accepted for publication in: The Astrophysical Journal 



\title{
X-ray Activity in the Open Cluster IC 4665
}

\author{
Mark S. Giampapa ${ }^{1}$, Charles F. Prosser ${ }^{2}$, and Thomas A. Fleming ${ }^{3}$
}

Accepted for publication in The Astrophysical Journal

1 National Optical Astronomy Observatories, National Solar Observatory, P.O. Box 26732, Tucson, AZ 85726-6732. The National Optical Astronomy Observatories are operated for the National Science Foundation by the Association of Universities for Research in Astronomy. E-mail: giampapa@noao.edu.

2 Harvard-Smithsonian Center for Astrophysics, 60 Garden St. MS-66, Cambridge, MA 02138. Current address: NOAO, P. O. Box 26732, Tucson, AZ 85726-6732. E-mail: cprosser@noao.edu.

3 Steward Observatory, University of Arizona, Tucson, AZ 85721. E-mail: tfleming@as.arizona.edu. 



\section{ABSTRACT}

We present the results of a joint ROSAT High Resolution Imager (HRI) and optical investigation of the open cluster IC 4665. The ROSAT data contains detections for 28 stellar sources in the field, including 22 cluster members and candidate members spanning the color range $-0.18 \leq(B-V)_{\mathrm{o}} \leq+1.63(\sim \mathrm{B} 3-\mathrm{M} 3)$. Upper limits are given for the remaining members (or candidate members) in the HRI field. Keck HIRES spectra have been obtained that yield radial and rotational velocity measures, respectively, for faint, low mass candidate members located within the field of the ROSAT HRI observation. In addition, photometry of possible optical counterparts to previously uncatalogued X-ray sources in the HRI field is presented.

The trends in X-ray properties with $(B-V)$ color in IC 4665 are found to be quite similar to that for other, more nearby young clusters such as the Pleiades and $\alpha$ Persei. In particular, a maximum in normalized X-ray luminosity of $\log \left(L_{x} / L_{b o l}\right) \simeq-3$ is observed, beginning in the color range of $(B-V)_{0}=0.7-0.8$. This is similar to the corresponding color range among Pleiades members, in agreement with the earlier estimate by Prosser \& Giampapa (1994) that the age of IC 4665 is similar to the age of the Pleiades. The correlation of rotation and X-ray emission levels is consistent with that in other young clusters.

Among the high mass stars in IC 4665 , five $\mathrm{B}$ stars are detected as X-ray sources. Of these, one is a spectroscopic binary while the remaining objects are apparently single stars. The level of intrinsic X-ray emission observed in the rapidly rotating $(v \sin i>200 \mathrm{~km}$ $\mathrm{s}^{-1}$ ), single $\mathrm{B}$ stars is consistent with an origin due to shock heating of the ambient medium by radiatively driven, rotationally enhanced winds. On the basis of these observations and the results for other clusters, we argue that observed levels of X-ray emission in high mass stars of $\log \left(L_{x} / L_{b o l}\right)>-6.0$ are likely due to intrinsic emission associated with the $\mathrm{B}$ star itself rather than emission from a late-type secondary. Finally, our results suggest that the initial angular momentum distributions and the subsequent evolution of rotation, $L_{x}$, and $\left(L_{x} / L_{b o l}\right)$ occur in practically identical fashions in clusters with ages $\$ 100 \mathrm{Myr}$. 



\section{INTRODUCTION}

The open cluster IC 4665 is among those nearby clusters for which there is significant membership information for both the high-mass and solar-type stars. The most recent studies include a proper motion membership survey by Prosser (1993), in which earlier work is summarized, followed by a radial velocity survey by Prosser \& Giampapa (1994). A summary of the properties of IC 4665 is given in Table 1; additional cluster names for IC 4665 and positional information are from Alter et al. (1970).

With an age similar to the Pleiades (Prosser \& Giampapa 1994), IC 4665 is distinguished from the other relatively nearby clusters surveyed by ROSAT by its high galactic latitude, corresponding to a distance from the Galactic plane of order $100 \mathrm{pc}$. The location of IC 4665 relative to the galactic plane is suggestive of an environment for star formation that was distinct from that in other open clusters. The kinematics of the IC 4665 cluster to reach large distances from the Galactic plane can indicate, for example, that its chemical composition may be intrinsically different from other clusters in the solar vicinity. The relationship between chemical composition and X-ray activity in solar-type stars has been recently discussed in an investigation of NGC2516 (Jeffries et al. 1997). A study of additional young clusters, such as IC 4665 , represents an important comparison to other clusters with extensive optical data bases that have also been the objects of intensive investigations with ROSAT. In order to study the X-ray properties of the members of IC 4665, we have obtained a deep ROSAT observation utilizing the High Resolution Imager (HRI). We also use the $\mathrm{X}$-ray observations presented in this investigation to assess the effectiveness of membership identification based on previous proper motion surveys. In particular, we utilize the HRI data to determine if any additional cluster members would be identified in X-rays which had been previously overlooked in optical studies.

\section{OPTICAL DATABASE}

Our optical database for IC 4665 is based upon the membership analysis in Prosser (1993), along with the radial velocity survey by Prosser \& Giampapa (1994). Rotation periods from photometric monitoring of solar-type IC 4665 members have been reported in Prosser (1993), Allain et al. (1996), and Prosser \& Grankin (1997). To further investigate the low-mass membership of IC 4665 and to supplement the X-ray observations, new observations of IC 4665 were conducted and are described below. The results of these additional observations have been combined with our previous results to form our optical database. 


\subsection{Spectral Type Determinations}

MK spectral classifications have been previously obtained for the brighter members of IC 4665 for the range B3-A5 (see Table 6 of Prosser 1993 and references therein). As the recent proper motion and radial velocity surveys successfully restricted the list of candidate members to only the most likely members, we wish to continue the spectral classifications begun by Abt and Levato (1975). Spectral types are useful for confirming cluster membership and estimating other stellar parameters (i.e. color excess, temperature, approximate radius and mass, etc.) which are utilized in detailed abundance studies (e.g., lithium) or in interpreting additional data for these stars (e.g., X-ray fluxes, rotation periods, etc.). A queue observing program utilizing the FAST CCD spectrograph at the Whipple Observatory 60-inch telescope was carried out during 1994 and 1996. Spectra were obtained using a $600 l \mathrm{~mm}^{-1}$ grating to provide $\sim 2000 \AA$ coverage at $1.49 \AA$ resolution at a central wavelength of $\sim 4400 \AA$. A series of standard stars classified on the MK system in the A5-K7V range were observed and are listed in Table 2. The spectral types are from Morgan et al. (1943), Johnson \& Morgan (1953), Keenan \& McNeil (1976), Keenan \& Pitts (1980), and Jacoby et al. (1984). A series of A7-K2III standards were observed to identify luminosity criteria. IC 4665 stars observed include those Kopff stars previously classified only on objective prism spectra (Table 6 in Prosser 1993); the radial velocity members identified in Prosser \& Giampapa (1994); and some of the brighter radial velocity ( $v_{\text {rad }}$ ) nonmembers. Since some of the $v_{\text {rad }}$ nonmembers of Prosser \& Giampapa (1994) could conceivably be binaries which are cluster members, spectral type information was obtained for some of these cases in order to ascertain if their spectral types might be consistent with cluster membership and therefore make them more likely targets for further radial velocity monitoring.

MK spectral classifications were obtained using a set of line strength ratios calibrated by the standard star observations: $\mathrm{H} \delta / \mathrm{Ca} 4226, \mathrm{H} \delta / \mathrm{Fe} 4045, \mathrm{H} \gamma / \mathrm{Fe} 4325$, and $\mathrm{H} \gamma / \mathrm{G}-$ band. Earlier than F0, only the $\mathrm{H} \delta / \mathrm{Ca} 4226$ ratio was considered, though none of our target stars were found to fall in this range. Measures of the $\mathrm{H} \gamma / \mathrm{Fe} 4325$ ratio were not attempted for stars earlier than $\simeq$ F6. For a particular target, the spectral types resulting from each index ratio were found to be consistent within a few subclasses in most cases, and an average, final MK spectral type for each star was estimated from the results of the measured indices and appearance of the spectrum. The MK spectral types for the stars in IC 4665 are given in Table 3 along with corrresponding indicators of membership status based on the spectral classification (i.e., "Y/Y?" = member/likely member, " $\mathrm{N}$ " = nonmember, and "?" = uncertain membership). All stars are found to be dwarfs, except for the nonmember P106 
which is classified as a giant. The spectral types for $\mathrm{K} 65, \mathrm{~K} 88$, and $\mathrm{P} 107$ are derived solely from the $\mathrm{H} \delta / \mathrm{Ca} 4226$ ratio, and might therefore be considered slightly less well determined. Among those Kopff stars with previous spectral type estimates (Table 6, Prosser 1993), the new FAST spectral types in Table 3 are more consistent with the positions of these stars in the color-magnitude diagram for the assumed cluster distance and reddening, thus resolving a previous discrepancy in this cluster.

Once considered as a cluster candidate, $\mathrm{P} 12$ can now be identified as a nonmember on the basis of its spectral type which is inconsistent with cluster membership for its observed color and assumed cluster reddening. $\mathrm{P} 12$ was noted as a rapid rotator $(v \sin i \simeq 70 \mathrm{~km}$ $\mathbf{s}^{-1}$ ) by Martín \& Montes (1997); the original Hamilton echelle observation reported in Prosser (1993) is considered to be in error. P12 appears therefore to be a rapidly rotating late-type field star which, as will be seen, is strongly detected in X-rays. Among observed radial velocity nonmembers, it is interesting to note that those proper motion candidates with the highest proper motion membership probabilities (P1, P33, P44, and P159) all have spectral types acceptable for membership - suggesting these stars as strong candidates for further study. On the other hand, the radial velocity nonmembers with low proper motion membership probabilities (K38=P6, P51, and P106), and the photometrically selected star P177, have spectral types which are either not consistent with cluster membership or are uncertain. Although photometry indicated that P20, P30, and P108 are likely not members, spectral types were nevertheless ascertained for these stars since, as will be seen, they were detected in X-rays. The resulting spectral types for these stars are not consistent with membership in IC 4665.

\subsection{Echelle Observations}

High-dispersion spectra of some $\mathrm{H} \alpha$ emission $\mathrm{M}$ dwarfs lying within the ROSAT HRI observation were obtained during 1994 Sept. 23-24 UT with the HIRES spectrograph (Vogt 1992) at Keck Observatory, Mauna Kea. The echelle spectrograph provided several orders including the $\mathrm{Li} \lambda 6707$ and $\mathrm{H} \alpha$ regions. A 0.861 arcsecond wide slit was used, providing a spectral resolution of $R \sim 45,000$. To determine radial and rotational velocities, an order in the $6405-6490 \AA$ range containing several strong $\mathrm{Fe} \& \mathrm{Ca}$ lines was chosen. As noted in Jones, Fischer, \& Stauffer (1996), the HIRES spectrograph is very stable; wavelength calibration was derived from thorium-argon comparison lamp exposures obtained at the beginning and end of each night. The flatfielded, extracted spectra were wavelength calibrated using the onedspec package in IRAF; radial velocity measures were subsequently obtained using the xcsao task (version 1.6) in the IRAF rvsao package. 
To serve as a check, the IC 4665 member P39 was also observed on each night that observations were obtained for the fainter $M$ dwarf candidates. Radial velocity analysis followed the same procedures as in Prosser \& Giampapa (1994). Due to the differences in spectral type between P39 and the fainter M dwarfs, standard stars appropriate for the target star(s) were adopted: for P39 the standard $v_{\text {rad }}$ stars were GL33, HD3765, HD29587, and HD154417, while for the fainter M dwarfs the standards used were GL176, GL654, GL873. The HD stars were taken from Latham \& Stefanik (1991), using the CfA radial velocities, while the Gliese stars are from Marcy \& Benitz (1989). Rotation velocities were derived using the same procedure as in Prosser \& Giampapa (1994); due to the differences in spectral types, two calibration template spectra were used - a spectrum of HR 996 (G5V, Soderblom et al. 1989) for P39 and a spectrum of GL654 (M3.5V) for the H $\alpha$ emission M dwarfs.

Equivalent width measures from the echelle spectra for the $M$ dwarfs that exhibit $\mathrm{H} \alpha$ emission are listed in Table 4. We estimate an uncertainty of about $10 \%$ in the equivalent width measures of the $\mathrm{H} \alpha$ emission line stength, as based primarily on the accuracy of the fitting of the local continuum. This method of error estimate follows the approach outlined by Worden, Schneeberger \& Giampapa (1981) in their measurements of the strengths of chromospheric lines in $\mathrm{M}$ dwarfs. Other factors that can affect the uncertainty include the $v \sin i$ of the star and intrinsic variability arising from stellar activity.

A summary of the $v_{\text {rad }} / v \sin i$ measures from the Keck HIRES observations is also given in Table 4. Members of IC 4665 are known to have radial velocities of $\sim-13 \mathrm{~km} \mathrm{~s}^{-1}$ (Prosser \& Giampapa 1994, Crampton et al. 1976, Morrell \& Abt 1991). The $v_{\text {rad }}$ measures for P39 are consistent with previous measures (Prosser \& Giampapa 1994), though a smaller $v \sin i$ value is found from these higher resolution, higher signal-to-noise CCD spectra. Among the $\mathrm{H} \alpha$ emission $\mathrm{M}$ dwarfs observed, most have $v_{\mathrm{rad}}$ measures consistent with membership, with nonmembership indicated only for P261. From comparison of the radial velocities derived for a given target star using different radial velocity standards (which were observed throughout each night), the accuracy of the $v_{\text {rad }}$ measures for the IC 4665 stars is on the order of $\pm 0.3 \mathrm{~km} \mathrm{~s}^{-1}$, though the error increases to $\pm 0.6 \mathrm{~km} \mathrm{~s}^{-1}$ for P257 - presumably as a result of its moderate rotation. Following Prosser \& Giampapa (1994), we estimate the accuracy of our $v \sin i$ measurements to be about $2-3 \mathrm{~km} \mathrm{~s}^{-1}$ in the range $10<v \sin i<30 \mathrm{~km} \mathrm{~s}^{-1}$, and increasing to $\sim 5 \mathrm{~km} \mathrm{~s}^{-1}$ for higher values of $v$ $\sin i$.

In addition to the Keck echelle observations, we also list in Table 4 the $v_{\mathrm{rad}} / v \sin i$ measures obtained for P97 and P117 using the KPNO 4m echelle and UV Camera with $79-63^{\circ}$ grating to yield $\sim 0.38 \AA$ ( 2 pix.) resolution spectra. For P97, the $v_{\text {rad }} / v \sin i$ 
measures were derived from a region of spectrum around $6400 \AA$, while for P117 the $v_{\text {rad }}$ measurement is derived from a portion of spectrum containing the $\mathrm{H} \alpha$ absorption feature - no reliable $v \sin i$ measurement could be obtained for P117 due to low signal-to-noise. As with the Keck observations, five/six radial velocity standard star observations each night were utilized to obtain an average $v_{\text {rad }}$ measure for the IC 4665 stars. The resulting radial velocities for P97 \& $\mathrm{P} 117$ clearly indicate nonmembership; from comparison using different radial velocity standards, the accuracy of the KPNO $v_{\text {rad }}$ measure for P97 is \pm 2 $\mathrm{km} / \mathrm{s}$, while that for $\mathrm{P} 117$ using the $\mathrm{H} \alpha$ feature is $\pm 6 \mathrm{~km} / \mathrm{s}$. Both $\mathrm{P} 97$ and P117 show $\mathrm{H} \alpha$ in absorption. The $v \sin i$ estimate for $\mathrm{P} 97$ was determined from comparison with a calibration template spectrum of the radial velocity standard HD3765 (K5).

The $\mathrm{H} \alpha$ profiles of the $\mathrm{M}$ dwarfs observed by Keck are illustrated in Fig. 1. The moderate rotation observed for $\mathrm{P} 257$ is reflected in its broadened $\mathrm{H} \alpha$ emission profile. The strong $\mathrm{H} \alpha$ emission observed in P230 exhibits a 'pedestal' or broadened wing component in its profile that is not evident in the other narrow-lined stars. This phenomenon has been observed in similar high-dispersion echelle observations of young stars in the Pleiades and IC2391/IC2602 clusters (Stauffer et al. 1997, Jones, Fischer \& Stauffer 1996). The likely origin of this feature has been attributed to flare or microflare events. In fact, the $\mathrm{H} \alpha$ equivalent width from the Keck echelle observation is twice as large as the previous measure from low-dispersion spectra (see Prosser 1993; his Table 5), in accordance with the flare hypothesis. However, it is also possible that the additional pedestal seen in the echelle data may not have been resolved from the surrounding spectrum in the earlier lower dispersion spectrum, resulting in the lower equivalent measurement given by Prosser (1993).

The Keck echelle spectra were also examined for the presence of the $\mathrm{Li} \lambda 6707$ feature. For the G0V star P39, an obvious $\mathrm{Li}$ absorption line is seen, with an equivalent width on the order of $170 \mathrm{~m} \AA$. Among the dMe stars, no significant feature is seen and an upper limit of $150 \mathrm{~m} \AA$ to the $\mathrm{EW}$ is estimated, with a higher upper limit of $300 \mathrm{~m} \AA$ assigned to $\mathrm{P} 261$ due, in part, to the lower $\mathrm{S} / \mathrm{N}$ level for this star. The lack of detectable lithium and the estimated upper limits are consistent with the results obtained for similar low mass $M$ dwarf members of the Pleiades (Jones, Fischer \& Stauffer 1996). 


\section{X-RAY OBSERVATIONS}

\subsection{ROSAT HRI Data}

Our present HRI observation of IC 4665 consists of a $76.9 \mathrm{ksec}$ exposure obtained at a position near the cluster center; details of the observation are given in Table 5. The location of the HRI field was chosen so as to include IC 4665 members extending over a range of mass, particularly solar-type members from Prosser \& Giampapa (1994) and a selection of the $\mathrm{H} \alpha$ emission $\mathrm{M}$ dwarfs found in the cluster region (see Table 5 and Fig. 5 in Prosser 1993). The data were analyzed using EXSAS routines running under MIDAS; the two separate observations obtained in 1995 and 1996 were combined directly into one observation since no significant offset in X-ray positions was found between the separate A \& B observations.

LOCAL \& MAP detections in EXSAS were run to create a provisional source list. This list was used as an input list to the maximum likelihood (ML) detection algorithm (Cruddace et al. 1988) to obtain a list of sources having ML $>5$. The source detections provided by EXSAS were visually examined in order to establish the reality of weaker sources. While most sources are identifiable at the ML $>7$ level, some sources with $5<\mathrm{ML}$ $<7$ were retained, particularly in cases where a star in our optical catalogue was known to be at the $\mathrm{X}$-ray position.

In Table 6 we list those catalogued stars which were detected in X-rays in our observation. The table lists star name, available optical information, the $(\alpha, \delta)$ offsets between $\mathrm{X}$-ray and optical positions (in the sense X-ray - optical), followed by the corresponding $\mathrm{X}$-ray information, luminosities and additional notes. The X-ray and optical positions all agree to within 15 arcsec or better. There are 253 catalogued stars in our IC 4665 database which fall within the HRI observation. For the $\sim 43 \mathrm{X}$-ray sources detected, one may estimate the number of chance alignments or matches between $\mathrm{X}$-ray sources and catalogued stars by multiplying the number of X-ray sources by the fraction of catalogued stars falling within a given distance from the X-ray position (following Randich et al. 1995). For matches within 15 arcsec from the $\mathrm{X}$-ray position, the number of chance alignments is on the order of $\sim 1.5$. Most of the stars in Table 6 lie within 10 arcsec from the $\mathrm{X}$-ray position; the corresponding number of chance alignments for this smaller radius is estimated to be less than one.

In order to convert the count-rates to observed X-ray fluxes and, eventually, X-ray 
luminosities, we utilized the cluster parameters in Table 1 and a conversion factor (CF) corresponding to a hydrogen column density of $\log \mathrm{N}_{H}\left(\mathrm{~cm}^{-2}\right)=21.0$ (Bohlin et al. 1978) along with the assumption of a Raymond-Smith plasma of temperature $k T=1.0 \mathrm{keV}$. The corresponding CF, based on the HRI calibration data given in Table 11.4 of the RMD (ROSAT Mission Description), is CF $=7.63 \times 10^{-11} \mathrm{erg} \mathrm{cm}^{-2} \mathrm{cnt}^{-1}$. The assumption of $k T=1.0 \mathrm{keV}$ is based on the results of Gagné, Caillault \& Stauffer (1995) who found that $k T=1.0 \mathrm{keV}$ was an accurate representation of the composite PSPC spectra they constructed from their observations of young, active dwarf stars in the Pleiades. In particular, Gagné et al. (1995) find that the emission measure of the hot $\left(\sim 10^{7} \mathrm{~K}\right)$ component exceeds the emission measure of the soft component in most cases. This hot component is characterized by $k T \sim 1.0 \mathrm{keV}$ in the composite spectra of G-M dwarfs constructed by Gagné et al., and slightly less than this value for $\mathrm{F}$ dwarfs. In addition, the higher extinction ( $\sim 5$ times higher than that of the Pleiades) associated with the greater distance of IC 4665 will tend to weight the present X-ray observations toward the harder component.

In the particular case of the B-star detections, we considered the possibility of UV contamination arising from the UV sensitivity of the ROSAT HRI. We adopted the scaling given by Schmitt et al. (1993) to find, at its maximum, a UV count rate of $\sim 10^{-4} \mathrm{ct}$ $\mathrm{s}^{-1}$ for $\mathrm{K} 62$ and less for the other detected high mass members. In particular, the ratio between the observed HRI count rate and the calculated UV rate ranges between $\sim 3-62$. We therefore conclude that the observed HRI counts in the detected high mass members are dominated by $\mathrm{X}$-ray emission rather than photospheric UV radiation.

In order to calculate the parameter $\left(L_{x} / L_{b o l}\right)$, bolometric corrections must be applied for the early-type and the later-type stars. Bolometric corrections $\left(\mathrm{BC}_{V}\right)$ were obtained from Harris $(1963$, his Table 7 ) for stars with $(\mathrm{B}-\mathrm{V}) \leq 1.20$ (approximately spectral type $\mathrm{K} 5$ and earlier), while $\mathrm{BC}_{\mathrm{I}}$ values were obtained from Monet et al. (1992, eqn. 6) for latertype stars. Solar values of $\log \mathrm{L}_{\mathrm{bol}}=33.583$ and $\mathrm{M}_{\mathrm{bol}}=4.76$ (Allen 1976) were assumed. For nonmember stars in Table 6 , the $\mathrm{L}_{\mathrm{X}}, \mathrm{L}_{\mathrm{bol}}$, and $\mathrm{B}-\mathrm{V}_{\mathrm{o}}$ values are provided only for comparison purposes as if they were really cluster members. Finally, we assume uniform extinction across our HRI field.

For those catalogued stars considered to be members and candidate members ( $Y / Y$ ?) or undecided (?), and which were not detected in X-rays (i.e., $\mathrm{ML}<7$ ), upper limits in the flux corresponding to a confidence level of $99.7 \%$ were determined; these values are listed in Table 7. For those stars in Table 6 with $\mathrm{ML}<7$, we provide upper limits in Table 7 should these sources later prove to be spurious. For stars with no measured B-V color, the $\mathrm{B}-\mathrm{V}_{\mathrm{o}}$ value in Table 7 has been estimated using the observed $\mathrm{V}-\mathrm{I}_{\mathrm{K}}$ color and the $\mathrm{B}-\mathrm{V}$ vs. $V-I_{K}$ relation in Prosser et al. (1996). Because of the increasing measurement errors 
in the HRI field for off-axis positions greater than 18 arcminutes, we regard the upper limits in Table 7 for such stars as less reliable. Therefore, in the discussion of these results, we will utilize only those upper limits determined for objects with off-axis positions of less than 18 arcminutes.

\section{RESULTS AND DISCUSSION}

We exhibit in Fig. $2 \log L_{x} v s$ intrinsic (B-V) color for detections of members and candidate members (i.e., "Y", "Y?" \& "?") from Table 6, and upper limits from Table 7 for members $(\mathrm{Y})$ and candidate members ( $\mathrm{Y}$ ?). Inspection of Fig. 2 reveals stellar X-ray sources spanning the color range $-0.17 \leq(B-V)_{\mathrm{o}} \leq+1.63$ ( B3 - M3). The general features of Fig. 2 also include a rise in $L_{x}$ beginning near F0 which is widely interpreted as evidence for the onset of outer convection zones and dynamo activity, analogous to that seen in the Sun. The observed X-ray emission in the cool stars reaches a maximum at $\sim$ G0 and then declines toward later spectral types. The decline in $L_{x}$ toward lower masses is a general feature of the X-ray properties of late-type stars, both in the field (Fleming et al. 1993) and in young clusters (Caillault 1996 and references therein). Fleming (1988) and Fleming et al. (1993) attribute this trend to the decline in stellar surface area, at least in the case of the most X-ray active stars where the surfaces may be entirely covered, or "saturated", with magnetic regions that are the sources of X-ray emission.

In Fig. 3 we show $\log \left(L_{x} / L_{b o l}\right)$ vs $(B-V)_{o}$ color for the same IC 4665 stars given in Fig. 2. The parameter $\left(L_{x} / L_{b o l}\right)$ is a normalized quantity that permits intercomparison among different spectral types. It is also interpreted as representative of the efficiency of coronal heating in late-type stars (Mullan 1984).

The general features in Fig. 3 include a rise from $\left(L_{x} / L_{b o l}\right) \sim 10^{-7}$ among the hot stars to a maximum level of $\left(L_{x} / L_{b o l}\right) \sim 10^{-3}$, possibly as early as $(B-V)_{0} \sim 0.7$. 0.8 , and certainly by $(B-V)_{0} \sim 0.9$. The latter has been referred to as the so-called 'saturation level' by a number of authors in studies of the X-ray properties of cluster members (Stauffer et al. 1994 [Pleiades]; Randich et al. 1996 [ $\alpha$ Per]; Stauffer et al. 1997 [IC 2602/IC 2391]). We note that prior studies of active, short-period binary systems in the field revealed saturation in the fluxes of far UV transition region lines (Vilhu \& Rucinski 1983; also see Vilhu 1984). The level of saturation is not due to radiative transfer effects; rather, it is considered a real property that is a manifestation of the underlying dynamo mechanism. The structures that give rise to the $\mathrm{X}$-ray emission are likely compact

regions (e.g., Giampapa et al. 1996; Schmitt \& Kürster 1993) since extended structures would be characterized by a volume filling factor that could yield values of $\left(L_{x} / L_{b o l}\right)$ that substantially exceed the observed maximum. 


\subsection{The High Mass Stars}

Our HRI field contains several high-mass members of IC 4665, including several detected B stars. A summary of the observed properties of the bright cluster members is given by Prosser (1993; see his Table 6). Using the membership information from Prosser (1993), we summarize in Table 8 the spectral type and X-ray data for the B and A star members and candidate members. Those stars not contained within the present HRI observation are noted in Table 8 with the term 'beyond field'. The observed X-ray emission among IC 4665 stars in Table 8 is comparable to that seen in similar high-mass stars in other clusters. While many chemically peculiar (CP) stars may be expected to have detectable levels of X-ray emission (see the discussion in Jeffries et al. 1997), the Hg-Mn star $\mathrm{K} 76 \mathrm{~A}$ is a non-magnetic $\mathrm{CP}$ star and is not necessarily expected to be an intrinsic X-ray source. Additional A-type members (Prosser 1993, his Table 6) not listed in Table 8 are generally outside of the field of the present HRI observation, though an upper limit has been determined for K66 (see Table 7).

A dispersion of $\sim 1.5 \mathrm{dex}$ in $\log \left(L_{x} / L_{b o l}\right)$ is seen for stars hotter than A0. Previous Xray studies of young clusters revealed similar $\sim 1.5$ dex dispersions in the X-ray properties among the high mass members (e.g, Randich et al. 1995 [IC2602]; Patten \& Simon 1993 [IC 2391]). We note, however, that the lower sensitivity limit of $\log \left(L_{x} / L_{b o l}\right) \sim-7.6$ for the high mass stars in the (more nearby) cluster IC 2391 would suggest that the actual range in $\log L_{x}$ among high mass members in IC 4665 is greater than indicated by our present data-set. This is in contrast to prior investigations that have quantitatively characterized the X-ray emission in young $\mathrm{B}$ stars by $\mathrm{L}_{x} \approx 10^{-7} \mathrm{~L}_{\text {bol }}$ (Schmitt et al. 1993). In the field, however, the X-ray properties of B stars are quite different. In particular, Cassinelli et al. (1994) find that $\left(L_{x} / L_{b o l}\right)$ declines sharply from $\sim 10^{-7}$ to $\sim 10^{-9}$ in the nearby, mainsequence B stars. Furthermore, the detected X-ray emission is characterized by relatively low source temperatures as inferred from the soft PSPC spectra. As Cassinelli et al. point out, B stars such as these would be difficult to detect at hydrogen column densities of $\log \mathrm{N}_{h}>20 \mathrm{~cm}^{-2}$. Hence, the $\mathrm{B}$ star sources in IC 4665 (where $\log \mathrm{N}_{h}=21 \mathrm{~cm}^{-2}$ ) must have relatively harder spectra in addition to the greater range in X-ray emission as compared to their counterparts in the field.

The X-ray emission observed in B stars is generally attributed to either intrinsic emission arising from shock heating of the surrounding medium by a high velocity, radiatively driven wind or to the presence of an active, late-type companion. For example, Meurs et al. (1992) suggest that the high $L_{x} / L_{\text {bol }}$ ratios deduced for some of the late-B stars in the ROSAT All-Sky Survey are due to the presence of late-type, binary companions. 
We see from inspection of Fig. 2, combined with the published X-ray data-sets for the Pleiades and $\alpha$ Per (see Fig. 4), that the maximum, non-flare X-ray luminosities of the late-type members of young clusters is $\log L_{x} \approx 30.5$. Adopting this value leads to an estimate of $\log \left(L_{x} / L_{b o l}\right) \lessgtr-6.0$ for mid-B stars if the X-ray emission is due entirely to a late-type companion. It would therefore seem unlikely that the observed $\mathrm{X}$-ray emission in high mass stars at levels exceeding $\log L_{x} / L_{b o l} \sim-6.0$ is due to a late-type companion. Moreover, the Morrell \& Abt (1991) survey of binaries in IC 4665 shows that the mass ratio of binary systems among the high mass stars is of order unity. Therefore, it would seem unlikely that the presence of an active, late-type companion is the origin of strong $\mathrm{X}$-ray emission in the high mass stars.

According to Morrell \& Abt (1991), the detected B stars in Table 8 exhibit constant radial velocities, with the exception of K49 which is a SB1 system. Since the secondary is not visible in the spectrum, it is presumably a low mass star. We can utilize the more complete X-ray data-set available for the Pleiades (since IC 4665 and the Pleiades are approximately the same age) and the observed value of $\log \mathrm{L}_{x}=29.28$ for $\mathrm{K} 49$ to infer that the companion is a late F - early K dwarf (see Fig. 12 in Stauffer et al. 1994).

The remaining, ostensibly single, high mass $\mathrm{X}$-ray sources include two objects, K73 and $\mathrm{K} 58$, with $\log \left(L_{x} / L_{b o l}\right)>-6.0$. These stars are rapid rotators with $v \sin i>200 \mathrm{~km}$ $\mathbf{s}^{-1}$. Thus, rotationally enhanced winds, such as those discussed by Poe \& Friend (1986) and Marlborough (1987), may play a role in the ultimate origin of the relatively strong $\mathrm{X}$-ray emission exhibited by these objects. The relatively slowly rotating $\mathrm{B}$ star, $\mathrm{K} 62$, is characterized by a low value of $\log \left(L_{x} / L_{b o l}\right)=-7.05$. However, K64 with a similarly high value of $v \sin i$ exhibits a more intermediate level of $\mathrm{X}$-ray emission among the high mass stars of IC 4665 with $\log \left(L_{x} / L_{b o l}\right)=-6.78$. Interestingly, the field star sample considered by Cassinelli et al. includes rapid rotators $\left(v \sin i>200 \mathrm{~km} \mathrm{~s}^{-1}\right)$ with quite low values of relative X-ray emission, i.e., $\log \left(L_{x} / L_{b o l}\right) \lesssim-7--9$. Apparently, there is a qualitative difference in the X-ray properties between the $\mathrm{B}$ stars in young clusters and $\mathrm{B}$ stars in the field. In brief summary, we attribute the strong $\mathrm{X}$-ray emission at levels exceeding $\sim-6.0$ in $\log \left(L_{x} / L_{b o l}\right)$ to an intrinsic property of the star and its interaction with the surrounding medium, perhaps through a rotationally enhanced wind.

The relatively lower number of detections in the A0 - F0 range is consistent with the claimed absence of $\mathrm{X}$-ray emission from stars without either outer convection zones or high velocity winds. In particular, the A stars appear to be X-ray quiet (Schmitt 1997); $\mathrm{X}$-ray emission that has been detected in early-to mid-A stars is generally attributed to the presence of a late-type companion (e.g., Schmitt et al. 1985; Schmitt \& Kürster 1993). 
We note that while a radial velocity survey of the B star members of IC 4665 has been performed (Morrell \& Abt 1991), a comparable survey of the A-type members has not yet been conducted. Hence, our understanding of binarity among the cluster A stars is limited. Apparently single, late-A stars in the field have been detected as X-ray sources (Simon et al. 1995). These objects likely represent the demarcation on the main sequence for the onset of outer convection (e.g., Wolff et al. 1986).

\subsection{The Low Mass Stars}

An intercomparison with other young clusters can yield further insight on the X-ray properties of the cool stars in IC 4665. In Fig. 4 we plot $\log L_{x}$ vs B-Vo for detected sources that are members of the Pleiades (Stauffer et al. 1994) and $\alpha$ Per (Randich et al. 1996), respectively. It is evident from Fig. 4 that the detected sources in IC 4665 follow the same trends with color as the other two clusters. A notable exception occurs at $(B-V)_{0} \sim 1.5$ where several objects in IC $4665-\mathrm{P} 232, \mathrm{P} 257, \mathrm{P} 270$, and P315 - exhibit levels of emission that are greater than that seen in either the Pleiades or $\alpha$ Per at this color. These particular objects may therefore not be members of IC 4665. Rather, they may be foreground dMe stars with true values of $L_{x}$ that are lower than would be deduced by assuming these objects are at the distance of IC 4665. In fact, P232, P257, P270 and P315 all lie $\sim 1 \mathrm{mag}$ above what may be considered the single star sequence in the $V$ vs $\left(V-I_{K}\right)$ color-magnitude diagram for IC 4665 (see Prosser 1993; his Fig. 5). This serves as an example of how X-ray observations can reveal possible nonmembers among photometrically selected candidates. Further observations are needed to finally confirm the membership status of these specific stars.

We have also compared the distribution of X-ray emission with color between IC 4665 and that for the even younger clusters IC 2391/2602 [Patten \& Simon 1993 (IC 2391); Randich et al. 1995 (IC 2602)], each with an age $\sim 35 \mathrm{Myr}$. As in the case of the comparison with the Pleiades and $\alpha$ Per, the observed trends of $\log L_{x}$ with color are similar for both clusters. Again, near $(B-V)_{\circ} \sim 1.5$, the suspected non-members in the IC 4665 field appear to exhibit higher levels of X-ray emission than the late-type members of IC $2391 / 2602$.

In Fig. 5 we include for comparative purposes the detected sources in IC 4665 along with the detections in $\alpha$ Per and the Pleiades, respectively. The values of $\log \left(L_{x} / L_{b o l}\right)$ for $\alpha$ Per are taken from Randich et al. (1996) while those for the Pleiades are given by Stauffer et al. (1994). ${ }^{4}$ While the IC 4665 observations are limited due to fewer stars,

4 We note in passing that the published upper limit of $\log \left(\mathrm{L}_{\mathrm{X}} / \mathrm{L}_{\text {bol }}\right)<-4.91$ for HII 
the distributions indicate that the $\log \mathrm{L}_{\mathrm{X}} / \mathrm{L}_{\mathrm{bol}} \simeq-3$ saturation level is reached at a similar color and spectral type in both the Pleiades and IC 4665. In IC 4665, the onset of saturation occurs in the color range $(B-V)_{0} \simeq 0.7-0.8$. which, in turn, is similar to the Pleiades where saturation begins at $(B-V)_{\circ} \simeq 0.75$. Previous investigations have shown that the color at which this maximum first occurs increases with increasing age of the cluster. For comparison we list in Table 9 the approximate colors where the onset of the saturation level of $\log \left(L_{x} / L_{b o l}\right) \sim-3$ has been observed in other clusters. The increasingly redder color (i.e., lower mass) at which saturation is attained in progressively older clusters is regarded as a manifestation of the mass-dependent rate for rotational spin down. In particular, the time scale for spin down via magnetic braking appears to increase with decreasing mass. Thus, the observed X-ray activity in IC 4665 provides further evidence that its age is similar to that of the Pleiades, as postulated by Prosser \& Giampapa (1994).

Inspection of Fig 5 reveals that the observed X-ray activity levels of the dwarf $\mathrm{M}$ stars in IC 4665 , the Pleiades and $\alpha$ Per extend above the $\log \left(\mathrm{L}_{\mathrm{X}} / \mathrm{L}_{\mathrm{bol}}\right) \sim-3$ saturation level seen for $G$ and $K$ dwarfs. The higher, relative levels of coronal activity in the $M$ dwarfs could be due to: 1) ROSAT is simply detecting those $M$ dwarfs which are flare-active at the time of observation, or 2) the -3 saturation level may not apply to the $\mathrm{M}$ dwarfs. Currently, no specific calculation has indicated why the activity among $\mathrm{G}$ and $\mathrm{K}$ dwarfs reaches a plateau at $\log \mathrm{Lx}_{\mathrm{X}} / \mathrm{L}_{\mathrm{bol}} \simeq-3$, and it may be that the changing physical structure in lower mass stars can lead to higher levels of coronal activity.

\subsubsection{The Correlation Of Coronal Activity With Rotation}

We illustrate in Fig. 6(a) the correlation between X-ray activity and rotation $(v \sin i)$ in the late-type stars. In Fig. 6(a) we see that $\log L_{x}$ exhibits an increasing trend with increasing rotational velocity. We also include for comparative purposes the relation for $L_{x}$ vs rotation from Pallavicini et al. (1981) inferred for F - M field stars, namely, $\log L_{x}$ $=27+2 \log (v \sin i)$. The empirical relationship between the normalized quantity $\log$ $\left(L_{x} / L_{b o l}\right)$ and rotational velocity is illustrated in Fig. 6(b). In view of the limited data, we do not attempt a functional fit. We note that the saturation plateau at $\left(L_{x} / L_{b o l}\right) \sim$ $10^{-3}$ begins to appear in stars with projected rotational velocities as low as $10 \mathrm{~km} \mathrm{~s}^{-1}$, and

2462 given by Stauffer et al. is incorrect and apparently results from an erroneous estimate of $\mathrm{L}_{\mathrm{bol}}$. A value of $\log \left(\mathrm{L}_{\mathrm{X}} / \mathrm{L}_{\mathrm{bol}}\right)<-4.35$ for HII 2462 has been used here. This correction becomes important for the accurate assessment of both the range and the lower limit of $\mathrm{X}$-ray activity observed in the solar-type stars in the Pleiades. 
definitively by $v \sin i \sim 20 \mathrm{~km} \mathrm{~s}^{-1}$. The so-called saturation itself is presumably related to a maximum efficiency in dynamo action that results in a filling factor of X-ray emitting structures on the stellar surface of order unity. In contrast to the absolute level of emission $\left(L_{x}\right)$, no further increase in $\left(L_{x} / L_{b o l}\right)$ occurs at rotational velocities exceeding $20 \mathrm{~km} \mathrm{~s}^{-1}$. We note, parenthetically, that the cluster member P27 $\left(B-V_{0}=0.59\right)$ is not detected at a relatively high upper $\operatorname{limit}$ of $\log L_{x}=30.00$ or $\log \left(L_{x} / L_{b o l}\right)=-3.73$, although it has a high rotational velocity of $v \sin i \simeq 33 \mathrm{~km} \mathrm{~s}^{-1}$. The measured X-ray flux limit may have been affected by its large off-axis position and/or otherwise influenced in some unknown manner by the fact that P27 is considered to be a SB1 system (Prosser \& Giampapa 1994).

We further examine the relationship between coronal X-ray emission in IC 4665 and dynamo parameters in Fig. $7(\mathrm{a})$ by utilizing the Rossby number $\left(R_{0}\right)$. This hydrodynamic parameter incorporates both rotation and convection, the key processes in dynamo action, in the form of a ratio between rotation period $\left(P_{\text {rot }}\right)$ and convective turnover time $\left(\tau_{c}\right)$. We compute the Rossby number for the G-M type cluster members for which we have rotation periods by adopting the relation between convective turnover time and $(B-V)$ color given by Noyes et al. (1984). We supplement these data with upper limits in rotation periods for those late-type stars in IC 4665 for which we have values of $v \sin i$. Stellar radii were estimated from $(B-V)_{0}$ colors and data given by Allen (1976). We include in Fig. 7(a) the linear relation between $\log \left(L_{x} / L_{b o l}\right)$ and $R_{0}$ found for stars in $\alpha$ Per by Randich et al. (1996). Our data appear consistent with a declining trend in normalized X-ray emission toward larger Rossby numbers. However, the few data combined with the upper limits in $\log \left(L_{x} / L_{b o l}\right)$ at the greatest extent of our range in $R_{0}$ precludes any further quantitative characterization. Since the Rossby number is a model-dependent quantity, we illustrate in Fig. $7(\mathbf{b})$ the relation between observed quantities, namely, $\log \left(L_{x} / L_{b o l}\right)$ and the rotation period. The data for IC 4665 are supplemented with estimates of rotation periods for stars with $v \sin i$ values only, as described above for Fig. 7(a). We include for comparative purposes the results given by Prosser \& Grankin (1997 and references therein) for G/Ktype members of $\alpha$ Per. As observed by Randich et al., $\left(L_{x} / L_{b o l}\right)$ appears to decline at rotation periods less than $\sim 10-12 \mathrm{hrs}$ in $\alpha$ Per. Currently, there are insufficient data to know whether this same trend is also present in IC 4665. We note that the early F dwarf, $\mathrm{K} 53$, appears underluminous in $\left(L_{x} / L_{b o l}\right)$ in Fig. $7(\mathrm{~b})$. By contrast, the location of K53 in Fig. $7(\mathrm{a})$ is somewhat more consistent with previous findings that the $\mathrm{X}$-ray emission levels in early F stars correlate well with Rossby number while no correlation is seen between rotation alone, and $L_{x}$ or $\left(L_{x} / L_{b o l}\right)$ for these objects with thin convection zones (Schmitt et al. 1985; Randich et al. 1996) 


\subsubsection{Chromospheric and coronal emission}

Finally, we illustrate in Fig. 8 the observed correlation between $\mathrm{H} \alpha$ equivalent width and coronal X-ray emission for the late-type stars in IC 4665 and the Pleiades. The $\mathrm{H} \alpha$ equivalent widths are taken from Table 4 and Prosser (1993) for IC 4665 while the $\mathrm{H} \alpha$ equivalent widths for Pleiades members are given by Stauffer \& Hartmann (1987). The basic form of this empirical relationship is the same in both clusters, i.e., the $\mathrm{H} \alpha$ absorption equivalent width fills in with (presumably) chromospheric emission, becoming weaker with increasing coronal activity. At $\log \left(L_{x} / L_{b o l}\right) \sim-3.5, \mathrm{H} \alpha$ appears in emission in the spectra of those IC 4665 stars for which we have both $\mathrm{H} \alpha$ and X-ray data. At the saturation level of $\log \left(L_{x} / L_{b o l}\right) \sim-3.0$, there is a large scatter in the $\mathrm{H} \alpha$ emission equivalent width.

The origin of the scatter in $\mathrm{H} \alpha$ strength may be of interest in the context of coronal and chromospheric structure. In particular, the saturation in X-ray emission is likely related to the compact nature of the X-ray structures, as suggested for the $\mathrm{M}$ dwarfs by Giampapa et al. (1996) and earlier, active (G) dwarfs by the results of Schmitt \& Kürster (1993). Hence, the upper limit or saturation in X-ray emission would be a function of stellar surface area, as pointed out by Fleming (1988). By contrast, the large scatter in chromospheric activity at the saturation level near $\log \left(L_{x} / L_{b o l}\right) \sim-3$ suggests that the region of $\mathrm{H} \alpha$ emission is not similarly constrained. That is, the structures which are the principal contributors to $\mathrm{H} \alpha$ emission may be more geometrically extended, in contrast to the relatively compact X-ray emitting regions. Flare activity in $\mathrm{H} \alpha$ would contribute to the scatter as well.

\section{3. $X$-ray Variability}

The two ROSAT observations of this HRI field, IC4665-A/IC4665-B, enables us to investigate the possibility of long-term $(\sim 1$ year) $\mathrm{X}$-ray variability in sources that are detected in at least one of the two observations. We identified potentially variable Xray sources by comparing the difference in X-ray counts between objects detected in both epochs to the quadratic sum of the error of the individual measurements, following Fleming et al. (1995). In the case of sources detected in one epoch but not the other, we compared the difference between the counts in the detection and the upper limit with the error in the detected source. Only one source, K56, was detected in both observations and was found to have a difference at the $3 \sigma$ level; the count rates, rate errors, and ML values are listed in Table 10.

We also list in Table 10 sources that are detected only in one observation where the difference between the detected count rate and the upper limit from the second observation 
exceeded the combined error. The ' $R$ ' $X$-ray sources in Table 10 will be described in the next section. We then constructed $\mathrm{X}$-ray light curves for these objects with an adopted bin size of $\sim 900 \mathrm{~s}$. Inspection of the X-ray light curve for the $3 \sigma$ source variable, K56 (A0; nonmember), did not show evidence of short-term variability within each separate observation. Moreover, we did not find evidence for variability similar to a flare-like outburst within the light curves of the remaining candidate variable $\mathrm{X}$-ray sources given in Table 10 . In particular, the count-rate appeared uniform to within the errors throughout the observing period in each epoch.

In the cases of K43, P230, P237, and R6, which were detected in IC4665-A, the count rates are less than the sensitivity limit we roughly estimate of $\sim 7 \times 10^{-4} \mathrm{cnts} \mathrm{s}^{-1}$ for the shorter-exposure, IC4665-B observation. Hence we would not expect to detect these objects in IC4665-B. Conversely, P248 and R12 should have been detected in IC4665-A. Similarly, the $\mathrm{M}$ dwarf $\mathrm{P} 232$ is strongly detected in the first epoch but not the second. In these cases, as with $\mathrm{K} 56$, we can only conclude that long-term variability on time scales of $\sim 1$ year can occur. We note that the dwarf $M$ stars that may be non-members $(\S 4.2)-$ P257, P270, and P315-in addition to P232, are characterized by relatively uniform X-ray light curves in our observations. However, the example of P232 suggests that these stars can exhibit long-term excursions in their X-ray emission between "high" and "low" states.

Finally, we examined the light curves for the two $B$ stars with the highest levels of X-ray emission, namely, K58 and K73. Again, the light curves were unremarkable and characterized by a uniform count-rate (to within the errors) during the course of observation in each epoch.

\subsection{Additional X-ray Sources}

Besides those X-ray sources associated with catalogued stars (Table 6), there were some additional $\mathrm{X}$-ray sources identified at the $\mathrm{ML}>5$ significance level in the HRI observation. These additional X-ray sources which were not matched to a catalogued star are given in Table 11. The sources are listed by a running ' $R$ ' number, followed by the $\mathrm{X}$-ray $(\alpha, \delta)$ coordinates, $\mathrm{ML}$ value, off-axis distance, count rate, and error in the count rate. Although these $\mathrm{X}$-ray sources are not necessarily associated with the IC 4665 cluster, for comparison purposes the corresponding X-ray luminosity for the distance of IC 4665 is given.

To identify possible optical counterparts to these X-ray sources, BVI CCD photometry was obtained during Nov. 1994 and Oct. 1995 using the 48 inch telescope of Whipple Observatory at Mt Hopkins, Arizona. For each night's observations, a set of extinction 
stars were monitored, along with standard stars from Landolt (1992), and some $\alpha$ Per members with photometry from Stauffer et al. (1985, 1989) and Prosser (1992). Each night's instrumental magnitudes were transformed onto a standard BV (Johnson) and I (Kron) system. Standards with Cousins-system V-I colors were transformed to the Kron V-I system using the transformation equations of Bessell \& Weis (1987). The standards were also used in determining the nightly aperture corrections applied to the photometry.

In Fig. 9, we provide finding charts at the $\mathrm{X}$-ray position for the ' $\mathrm{R}$ ' $\mathrm{X}$-ray sources in Table 11. Charts are approximately $3 \times 3 \mathrm{arcmin}$, North up and East to the left, and are extracted from a digitized scan of the Palomar Schmidt XE plate using the GASP software at STScI. The circles are centered at the position of the $\mathrm{X}$-ray detection and have a radius of $\sim 30$ arcsec. For each X-ray source, typically several stars within $\sim 30$ arcsec of the X-ray position were measured for photometry. As seen from Table 6, most optical counterparts - particularly for the stronger X-ray sources - may be expected to be found within $\sim 15$ arcsec from the X-ray position. Additional counterparts out to $\sim 30$ arcsec radius in Fig. 9 were measured as either secondary targets or for comparison purposes. Following the identifications in Fig. 9, the BVI photometry for the possible optical counterparts to the ' $R$ ' $X$-ray sources is given in Table 12. No photometry is available for stars in the region of sources R2 and R5. VI CCD images obtained during non-photometric conditions for us by R. Schild indicate that a faint red object is located near or at the X-ray position of R5. For the strong $\mathrm{X}$-ray source $\mathrm{R} 14$, we note the presence of a faint red companion to the bright star in Table 11 with a separation of only 3 arcsec. An MK spectral type of K5-K7 V has been derived for the brighter star from a spectrum obtained with the FAST spectrograph (section 2.1). Without additional data however, we cannot precisely determine whether the bright star or its faint red companion is the source of $\mathrm{X}$-ray emission.

\section{SUMMARY}

Our joint X-ray and optical investigation of IC 4665 represents a further contribution to the recent $R O S A T$ studies of young clusters. At a galactic latitude of +17 degrees, the location of IC 4665 suggests that its formation history in the galactic environment may be uniquely different from other low galactic latitude clusters. Our ROSAT investigation of IC 4665 is complemented by a significant and expanding optical data base which has been supplemented herein with new Keck HIRES spectra of several faint, low mass members. In addition, photometry of possible optical counterparts to previously uncatalogued sources is given in this investigation.

The comparison of the detected sources and upper limits for members and candidate 
members of IC 4665 with that of other clusters, including the Pleiades, $\alpha$ Per, and IC $2391 / 2602$, reveals considerable overlap and similarities in the distribution of X-ray properties with $(B-V)_{\circ}$ color. In all clusters, a maximum or "saturation" level in normalized $\mathrm{X}$-ray luminosity is attained at $\log \left(L_{x} / L_{b o l}\right) \sim-3$ in the low mass stars.

More specifically, the detected sources in IC 4665 follow the same trends in $L_{x}$ and $\left(L_{x} / L_{b o l}\right)$ with $(B-V)$ color as seen in other young clusters such as $\alpha$ Per, the Pleiades, and IC 2391/2602. These trends include a rise in both $L_{x}$ and $\left(L_{x} / L_{b o l}\right)$ in the late-type stars beginning near $\sim$ F0. This trend is associated with the onset of solar-like dynamo activity. A maximum or "saturation limit" of $\log \left(L_{x} / L_{b o l}\right) \sim-3$ is attained in IC 4665 in the color range $(B-V)_{0} \sim 0.70-0.8$ which, in turn, is similar to that found in the Pleiades (i.e., $\mathrm{B}-\mathrm{V}_{\mathrm{o}} \simeq 0.75$ ). We view this as further evidence that the ages of IC 4665 and the Pleiades are approximately the same, as concluded by Prosser \& Giampapa (1994).

The X-ray observations suggest that several photometrically selected $M$ dwarfs with $\mathrm{H} \alpha$ emission are actually non-members. However, the relatively enhanced X-ray emission in these stars, compared to those in the Pleiades and $\alpha$ Per at the same color, could also result from flare activity-though no evidence for flare-like outbursts was evident in an inspection of the X-ray light curves for both epochs of observation. Nevertheless, the results for the specific case of P232 imply that long-term ( $\sim 1$ year) variability that is characterized as a change in activity from a "high" to a "low" state can occur in these stars (independent of the question of membership).

The empirical correlation of rotation and X-ray emission in IC 4665 is similar to that of other young clusters, at least as seen within the limitations of the available data. The saturation level is certainly attained at rotational velocities of $v \sin i=20 \mathrm{~km} \mathrm{~s}^{-1}$ and, in IC 4665 , possibly by a rotational velocity as low as $\sim 10 \mathrm{~km} \mathrm{~s}^{-1}$. Finally, we note that in the emission line stars there is significant scatter in the $\mathrm{H} \alpha$ emission equivalent widths at $\log \left(L_{x} / L_{b o l}\right) \sim-3$. Apparently, $\mathrm{H} \alpha$ is not similarly "saturated" but may instead be dominated by emission from geometrically extended regions while the $\mathrm{X}$-ray emission arises primarily from surface regions that are relatively compact (Giampapa et al. 1996). Conversely, the Keck HIRES observations of $\mathrm{H} \alpha$ emission line profiles in objects such as P230 imply that flaring, which is more prominent in $\mathrm{H} \alpha$, could be the source of the large scatter in the strength of this feature at high normalized X-ray luminosities.

Among the 5 high mass $\mathrm{B}$ stars that are $\mathrm{X}$-ray sources, one source is a spectroscopic binary (K49). The level of emission for K49 is consistent with that of a late $\mathrm{F}$ - early $\mathrm{K}$ secondary though intrinsic X-ray emission in the B star primary is by no means excluded. The remaining sources are not radial velocity variables and are presumed to be single $B$ 
stars. Moreover, we argue that for values of $\left(L_{x} / L_{b o l}\right)>10^{-6}$, it is unlikely that the $\mathrm{X}$-ray emission is due to a late-type companion. Instead, $\mathrm{X}$-ray activity in high mass stars is attributed to shock heating of the ambient medium by a radiatively driven wind that may also be enhanced by rotation.

The similarity of X-ray properties between IC 4665 and other young ( $~ 30-100 \mathrm{Myr})$ clusters suggests that formation environment or formation history of open clusters is not reflected in the nature of stellar coronae, at least for clusters with solar-like metallicities. We therefore infer that, in clusters with ages $<100 \mathrm{Myr}$, the distributions of rotational velocities with color for the low mass stars later than about $(B-V)_{0}=0.6$ are similar.

This study was supported by NASA Grant Nos. NAG5-3238 (TAF); NAGW-2698 and NAG5-2203 (CFP); and an interagency transfer from NASA to the NOAO (MSG). The FAST spectra in IC 4665 were obtained by Perry Berlind during service observing and we gratefully acknowledge his diligence in obtaining the requisite observations for this program. The W.M. Keck Observatory is operated as a scientific partnership between the California Institute of Technology and the University of California. It was made possible by the generous financial support of the W.M. Keck Foundation. 


\section{References}

Allain, S., Bouvier, J., Prosser, C., Marschall, L.A., \& Laaksonen, B.D., 1996, A\&A, 305, 498

Allen, C.W. 1976, Astrophysical Quantities, 3rd ed. (Athlone, London)

Alter, G., Balázs, B., \& Ruprecht, J. 1970, Catalogue of Star Clusters and Associations, 2nd Ed. (Akadémiai Kiadó, Budapest)

Abt, H.A., \& Levato, H. 1975, PASP, 87, 849

Bessell, M.S., \& Weis, E.W. 1987, PASP, 99, 642

Bohlin, R. C., Savage, B. D., \& Drake, J. F. 1978, ApJ 224, 132

Caillault, J.-P. 1996 in Cool Stars, Stellar Systems and the Sun, eds. R. Pallavicini \& A. K. Dupree, ASP Conf. Series vol. 109, p. 325

Cassinelli, J. P., Cohen, D. H., MacFarlane, J. J., Sanders, W. T., \& Welsh, B. Y. 1994, ApJ, 421, 705

Crampton, D., Hill, G., \& Fisher, W.A. 1976, ApJ, 204, 502

Cruddace, R., Hasinger, G., \& Schmitt, J.H.M.M. 1988, in Proceedings of ESO Conference on Large Databases, edited by F. Murtagh (ESO, Garching), p 177

Fleming, T. A. 1988, Ph.D. thesis, University of Arizona, Tucson.

Fleming, T. A., Giampapa, M. S., Schmitt, J.H.M.M., \& Bookbinder, J. A. 1993, ApJ, 410,387

Fleming, T. A., Molendi, S., Maccacaro, T., \& Wolter, A. 1995, ApJS, 99, 701

Gagné, M., Caillault, J.-P., \& Stauffer, J. R. 1995, ApJ, 450, 217

Giampapa, M. S., Rosner, R., Kashyap, V., Fleming, T. A., Schmitt, J. H. M. M., \& Bookbinder, J. A. 1996, ApJ, 463, 707

Harris, D.L. 1963, in Basic Astronomical Data, edited by Strand. (University of Chicago, Chicago), p. 263

Jacoby, G.H., Hunter, D.A., \& Christian, C.A. 1984, ApJS, 56, 257

Jeffries, R.D., Thurston, M.R., \& Pye, J.P. 1997, MNRAS, in press

Johnson, H.L., \& Morgan, W.W. 1953, ApJ, 117, 313

Jones, B.F., Fischer, D.A., \& Stauffer, J.R. 1996, AJ, 112, 1562

Keenan, P.C., \& McNeil, R.C. 1976, An Atlas of the Spectra of the Cooler Stars (Ohio State University Press, Columbus)

Keenan, P.C., \& Pitts, R.E. 1980, ApJS 42, 541

Kopff, E. 1943, ANac, 274, 69

Landolt, A. 1992, AJ, 104, 340

Latham, D.W., \& Stefanik, R.P. 1991, in Reports in Astronomy, IAU Transactions XXIB (Kluwer, Dordrecht), p. 269 (CfA preprint No. 3316) 
Marcy, G.W. \& Benitz, K.J. 1989, ApJ, 344, 441

Marlborough, J. W. 1987, in Physics of Be Stars, eds. A. Slettebak \& T. P. Snow, p. 316 (Cambridge: Cambridge University Press)

Martín, E.L. \& Montes, D. 1997 A\&A 318, 805

Meurs, E. J. A. et al. 1992, A\&A, 265, L41

Monet, D.G., Dahn, C.C., Vrba, F.J., Harris, H.C., Peir, J.R., Luginbuhl, C.B, \& Ables H.D. 1992, AJ 103, 638

Morgan, W.W., Keenan, P.C., and Kellman, E. 1943, An Atlas of Stellar Spectra (University of Chicago Press, Chicago)

Morrell, N., \& Abt, H.A., 1991, ApJ, 378, 157

Mullan, D. J. 1984, ApJ, 282, 603

Noyes, R. W., Hartmann, L. W., Baliunas, S. L., Duncan, D. K., \& Vaughan, A. H. 1984, ApJ, 279, 763

Pallavicini, R., Golub, L., Rosner, R., Vaiana, G. S., Ayres, T., \& Linsky, J. L. 1981, ApJ, 248,279

Patten, B. M., \& Simon, T. 1993, ApJL 415, L123

Poe, C. H., \& Friend, D. B. 1986, ApJ, 311, 317

Prosser, C.F. 1992, AJ, 103, 488

Prosser, C.F. 1993, AJ 105, 1441

Prosser, C.F., \& Giampapa, M.S., 1994, AJ 108, 964

Prosser, C.F., \& Grankin, K. 1997, Center for Astrophysics Preprint No. 4539.

Prosser, C.F., Stauffer, J.R., Caillault, J.-P., Balachandran, S., Stern, R.A., \& Randich, S. $1995, \mathrm{AJ} 110,1229$

Prosser, C.F., Randich, S.R., Stauffer, J.R., Schmitt, J.H.M.M., \& Simon, T. 1996, AJ 112,1570

Randich, S., \&Schmitt, J.H.M.M. 1995, A\&A 298, 115

Randich, S., Schmitt, J.H.M.M., Prosser, C., \& Stauffer, J.R. 1995, A\&A 300, 134

Randich, S., Schmitt, J.H.M.M., Prosser, C., \& Stauffer, J.R. 1996, A\&A 305, 785

Schmitt, J.H.M.M., Golub, L., Harnden, F. R., Jr., Maxson, C. W., Rosner, R., \& Vaiana, G. S. 1985, ApJ, 290,307

Schmitt, J.H.M.M., Zinnecker, H., Cruddace, R., \& Harnden, F. R., Jr. 1993 ApJL, 402, L13

Schmitt, J.H.M.M. \& Kürster, M. 1993, Science, 262, 215

Schmitt, J.H.M.M. 1997, A\&A, 318, 215

Simon, T., Drake, S. A., \& Kim, P. D. 1995, PASP, 107, 1034

Soderblom, D.R., Pendleton, J., \& Pallavicini, R. 1989, AJ, 97, 539 
Stauffer, J.R., Hartmann, L.W., Burnham, J.N., and Jones, B.F. 1985, ApJ, 289, 247

Stauffer, J. R., \& Hartmann, L. W. 1987, ApJ, 318, 337

Stauffer, J.R., Hartmann, L.W., \& Jones, B.F. 1989, ApJ, 346, 160

Stauffer, J.R., Caillault, J.-P., Gagné, M., Prosser, C.F., \& Hartmann, L.W. 1994, ApJS 91,625

Stauffer, J.R., Hartmann, L.W., Prosser, C.F., Randich, S., Balachandran, S., Patten, B., Simon, T., \& Giampapa, M. 1997, ApJ, 479, 776

Stern, R.A., Schmitt, J.H.M.M., \& Kahabka, P.T. 1995, ApJ 448, 683

Vogt, S.S. 1992, in ESO Workshop on High Resolution Spectroscopy with the VLT (ESO, Garching), p. 223

Vilhu, O. \& Rucinski, S. 1983, A\&A 127, 5

Vilhu, O. 1984, A\&A, 133,117

Wolff, S. C., Boesgaard, A. M., \& Simon, T. 1986, ApJ, 310, 360

Worden, S, P., Schneeberger, T. J., \& Giampapa, M. S. 1981, ApJS, 46, 159 


\section{Figure Captions}

Fig. 1. H $\alpha$ line profiles in the spectra of the dMe stars observed with the Keck HIRES echelle.

Fig. 2. The distribution of $\mathrm{X}$-ray luminosity with color for members and candidate members in IC 4665 . The solid circles represent detections while the arrows denote upper limits. Approximate spectral types are indicated.

Fig. 3. The distribution of the normalized quantity $\log \left(L_{x} / L_{b o l}\right)$ with $\mathrm{B}-\mathrm{V}_{\mathrm{o}}$ color for IC 4665 stars displayed in Fig. 2. A dashed line is drawn at the saturation level log $\left(L_{x} / L_{b o l}\right) \simeq-3$ that has been observed in other young clusters.

Fig. 4. Comparison of the distribution of $L_{x}$ with $(B-V)$ color of stars in IC 4665 with members of (a) the Pleiades and (b) $\alpha$ Per.

Fig. 5. Comparison of the distributions in $\mathrm{L}_{X} / \mathrm{L}_{\text {bol }}$ among IC 4665 stars with (a) the Pleiades and (b) $\alpha$ Per. The figure indicates that the onset of the saturation level in solar-type stars occurs at a similar color for IC 4665 and the Pleiades.

Fig. 6. (a) The $\log L_{x}$ vs $\log (v \sin i$ ) distribution for dwarf $\mathrm{G} / \mathrm{K} / \mathrm{M}$ stars in IC 4665 with available $v \sin i$ measures. Stars with upper limits in $\mathrm{LX}_{\mathrm{X}}$ and/or $v \sin i$ are indicated with arrows. The linear relation is discussed in the text. (b) The correlation between $\log \left(\mathrm{L}_{\mathrm{X}} / \mathrm{L}_{\mathrm{bol}}\right)$ and rotation in IC 4665. The saturation level at $\log \left(\mathrm{L}_{\mathrm{X}} / \mathrm{L}_{\mathrm{bol}}\right) \sim-3.0$ is indicated by the dashed line.

Fig. 7. (a) $\log \left(\mathrm{L}_{\mathrm{X}} / \mathrm{L}_{\mathrm{bol}}\right)$ vs. the logarithm of the Rossby number $\left(P_{\text {rot }} / \tau_{c}\right)$ for IC 4665 stars. Upper limits in rotation periods have been estimated for those stars with measured $v \sin i$ values. Objects with upper limits in either rotation period or normalized X-ray luminosity are indicated with arrows. The dashed line is the approximately inverse relation inferred by Randich et al. (1996) for $\alpha$ Per stars in the regime where log $\left(P_{\text {rot }} / \tau_{c}\right) \geq-1.2$. (b) Late-type stars in IC 4665 with either observed rotation periods or upper limits estimated from $v \sin i$ measurements and estimates of the stellar radius . Stars in $\alpha$ Per with observed rotation periods are also displayed for comparison.

Fig. 8. The correlation between $\mathrm{H} \alpha$ equivalent width and $\mathrm{X}$-ray emission for stars in IC 4665 and the Pleiades, respectively.

Fig. 9. Finding charts for the additional $\mathrm{X}$-ray sources in Table 11. Fields shown are $3 \times 3$ arcmin, north up and east to the left. Catalogued stars $(\mathrm{K} / \mathrm{P})$ and stars measured for photometry are marked. 
TABLE 1. IC4665: General Information

\begin{tabular}{|c|}
\hline 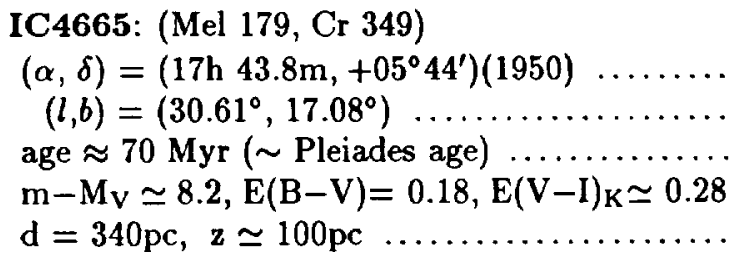 \\
\hline
\end{tabular}


TABLE 2. MK Spectral Type Standards

\begin{tabular}{cccc}
\hline \hline Star & Spt & Star & Spt \\
\hline 80 UMa & A5V & $\epsilon$ Boo & G8V \\
$\theta$ Cas & A7V & $\sigma$ Dra & K0V \\
$\epsilon$ Cep & F0V & SAO11483 & K4V \\
$\sigma$ Boo & F2V & 61 CygA & K5V \\
$\chi$ Dra & F6V & 61 CygB & K7V \\
$\xi$ Peg & F7V & $\gamma$ Boo & A7III \\
SAO11547 & F8V & $\gamma$ Her & A9III \\
$\beta$ Com & G0V & $\beta$ Del & F5III \\
$\lambda$ Ser & G0V & 84 Her & G2III \\
16 CygA & G3V & $\kappa$ Oph & K2III \\
16 CygB & G5V & & \\
& & & \\
\hline
\end{tabular}


TABLE 3. IC4665 MK Spectral Types

\begin{tabular}{cccccc}
\hline \hline Star & Spt & Mem & Star & Spt & Mem \\
\hline K34 & F5V & Y & P44 & G0V & Y \\
K37 & F2V & Y & P51 & F8V & $?$ \\
K38 & F3/F5V & Y? & P60 & G8V & Y \\
K48 & F3V & Y & P71 & K0V & Y \\
K53 & F2V & Y & P75 & G8V & Y \\
K57 & F1V & Y & P94 & ZK0V & Y \\
K65 & F0:V & Y & P100 & K0V & Y \\
K88 & F0:V & Y & P106 & K0III & N \\
P1 & F5V & Y & P107 & G2:V & Y \\
P12 & G8V & N & P108 & G8V & N \\
P19 & F8V & Y & P146 & K0V & Y \\
P20 & F6V & N & P150 & G5V & Y \\
P27 & G0V & Y & P155 & K0V & Y \\
P30 & G3V & N & P159 & F6V & Y \\
P33 & G0V & Y & P165 & K0V & Y \\
P38 & G0V & Y & P166 & K0V & Y \\
P39 & G0V & Y & P177 & G0V & N \\
& & & & & \\
\hline
\end{tabular}


TABLE 4. Keck/KPNO Echelle Observations

\begin{tabular}{|c|c|c|c|c|c|c|c|c|}
\hline Star & V & $V-I_{K}$ & Mem? & $\begin{array}{c}v_{\mathrm{rad}} \\
(\mathrm{km} / \mathrm{s})\end{array}$ & $\begin{array}{c}v \sin i \\
(\mathrm{~km} / \mathrm{s})\end{array}$ & $\begin{array}{c}\mathrm{H} \alpha \mathrm{EW} \\
(\AA)\end{array}$ & UT Date & $\begin{array}{c}\text { JD - } \\
2449600.0\end{array}$ \\
\hline \multicolumn{9}{|l|}{ Keck: } \\
\hline P39 & 12.93 & 0.69 & $\mathrm{Y}$ & -14.6 & 12 & (abs) & Sep 23, 1994 & 18.75217 \\
\hline$\ldots$ & $\cdots$ & & $\ldots$ & -13.7 & 12 & (abs) & Sep 24, 1994 & 19.76371 \\
\hline P230 & 17.57 & 2.60 & $\mathbf{Y}$ & -14.6 & 12 & 8.25 & Sep 23, 1994 & 18.82164 \\
\hline P248 & 17.92 & 2.49 & $\mathbf{Y}$ & -13.8 & $<10$ & 1.84 & Sep 23, 1994 & 18.85182 \\
\hline P257 & 17.14 & 2.60 & $\mathbf{Y}$ & -14.6 & 52 & 4.85 & Sep 24, 1994 & 19.83039 \\
\hline P261 & 19.31 & 3.18 & $\mathrm{~N}$ & -36.9 & $<10$ & 2.33 & Sep 24, 1994 & 19.78315 \\
\hline P265 & 17.20 & 2.29 & $\mathrm{Y} ?$ & -10.8 & $<10$ & 0.54 & Sep 23, 1994 & 18.77098 \\
\hline P270 & 17.27 & 2.57 & $\mathrm{Y} ?$ & -18.4 & $<10$ & 2.47 & Sep 24, 1994 & 19.80974 \\
\hline P336 & 17.37 & 2.29 & $\mathrm{Y}$ & -14.4 & 10 & 1.61 & Sep 23, 1994 & 18.79859 \\
\hline \multicolumn{9}{|c|}{ KPNO: } \\
\hline P97 & 14.93 & 1.33 & $\mathrm{~N}$ & +22 & $<10$ & (abs) & Nov 24, 1994 & 80.57465 \\
\hline P117 & 14.89 & 1.05 & $\mathrm{~N}$ & +52 & $\ldots$ & (abs) & Nov 21,1994 & 77.56568 \\
\hline
\end{tabular}


TABLE 5. ROSAT HRI Observations of IC4665.

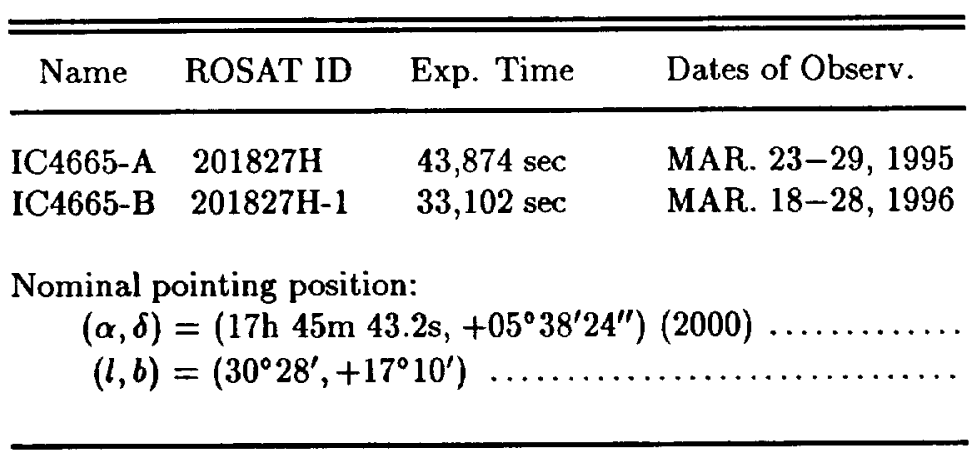


TABLE 6. IC4665 Stars Detected in X-rays

\begin{tabular}{|c|c|c|c|c|c|c|c|c|c|c|c|c|c|c|c|c|}
\hline Name & Mem & V & $B-V$ & $V-I_{K}$ & Spt & $\begin{array}{c}v \sin i \\
\left(\mathrm{~km} \mathrm{~s}^{-1}\right)\end{array}$ & $\begin{array}{l}\Delta \alpha \Delta \delta \\
\left({ }^{\prime \prime}\right)\left({ }^{\prime \prime}\right)\end{array}$ & ML & $\begin{array}{c}\text { Offax } \\
\text { (arcmin) }\end{array}$ & $\begin{array}{l}\text { Cnts } \\
\left(s^{-1}\right)\end{array}$ & $\begin{array}{c}\text { Err } \\
\left(s^{-1}\right)\end{array}$ & $\log L_{x}$ & $\log L_{b}$ & $\begin{array}{c}\log \\
\mathrm{L}_{\mathrm{X}} / \mathrm{L}_{\text {bol }}\end{array}$ & $\mathrm{B}-\mathrm{V}_{\mathrm{o}}$ & Notes \\
\hline K62 & $\mathbf{Y}$ & 6.87 & 0.00 & $\ldots$ & B3IV & 50 & $+2-7$ & 13.8 & 9.0 & 0.0004 & 0.0001 & 29.61 & 36.66 & -7.05 & -.18 & \\
\hline K58 & $\mathbf{Y}$ & 7.59 & 0.01 & ... & $\mathrm{B} 6 \mathrm{~V}$ & 200 & $+2-2$ & 592.1 & 4.8 & 0.0041 & 0.0003 & 30.64 & 36.28 & -5.64 & -.17 & \\
\hline K49 & Y & 7.69 & 0.02 & $\cdots$ & B6V & 25 & $-6+2$ & 6.5 & 5.2 & 0.00018 & 0.00007 & 29.28 & 36.24 & -6.96 & -.16 & SB1 \\
\hline K64 & $\mathbf{Y}$ & 7.35 & 0.02 & $\ldots$ & B5IV & 220 & $+4-3$ & 12.1 & 7.0 & 0.0004 & 0.0001 & 29.60 & 36.38 & -6.78 & -.16 & \\
\hline K73 & $\mathbf{Y}$ & 7.13 & 0.02 & $\ldots$ & B5IV & 210 & $+4-1$ & 88.9 & 16.5 & 0.0032 & 0.0003 & 30.53 & 36.47 & -5.94 & -.16 & \\
\hline $\mathrm{K} 43$ & $\mathbf{Y}$ & 9.08 & 0.17 & $\ldots$ & A1V & 185 & $+0+7$ & 5.1 & 6.7 & 0.0004 & 0.0001 & 29.67 & 35.29 & -5.62 & -.01 & \\
\hline K67NS & $\mathbf{Y}$ & 9.6 & $\ldots$ & $\ldots$ & $\mathrm{A} 2 \mathrm{~V}$ & 250: & $+3-2$ & 50.8 & 11.4 & 0.0013 & 0.0002 & 30.12 & 35.02 & -4.90 & 0.06 & VB $3^{\prime \prime}$ \\
\hline K53 & $\mathbf{Y}$ & 11.43 & 0.51 & $\ldots$ & F2V & 38: & $+5-4$ & 61.1 & 4.7 & 0.0008 & 0.0001 & 29.92 & 34.22 & -4.30 & 0.33 & \\
\hline P38 & $\mathbf{Y}$ & 12.42 & 0.74 & 0.77 & GOV & 67 & $+3-4$ & 169.4 & 12.9 & 0.0032 & 0.0003 & 30.52 & 33.81 & -3.29 & 0.56 & \\
\hline P60 & $\mathbf{Y}$ & 13.43 & 0.88 & 0.80 & G8V & 13 & $-11-6$ & 7.4 & 17.4 & 0.0010 & 0.0003 & 30.00 & 33.44 & -3.44 & 0.70 & \\
\hline P71 & $\mathbf{Y}$ & 13.68 & 0.92 & 0.91 & KOV & 17 & $+3+0$ & 83.8 & 11.0 & 0.0017 & 0.0002 & 30.24 & 33.35 & -3.11 & 0.74 & \\
\hline P100 & $\mathbf{Y}$ & 14.37 & 1.06 & 1.10 & K0V & 21 & $+4+0$ & 82.1 & 7.8 & 0.0012 & 0.0002 & 30.11 & 33.11 & -3.00 & 0.88 & \\
\hline P207 & $?$ & 15.50 & 1.24 & 1.33 & $\ldots$ & $\ldots$ & $+3-4$ & 29.5 & 10.0 & 0.0009 & 0.0002 & 29.95 & 32.76 & -2.81 & 1.06 & \\
\hline P290 & $?$ & 14.88 & 1.41 & 1.48 & $\ldots$ & ... & $+2-2$ & 45.5 & 7.1 & 0.0007 & 0.0001 & 29.84 & 33.10 & -3.26 & 1.23 & \\
\hline P232 & $?$ & 15.92 & 1.61 & 1.85 & $\ldots$ & $\ldots$ & $+3+0$ & 38.6 & 9.9 & 0.0009 & 0.0002 & 29.99 & 32.82 & -2.83 & 1.43 & \\
\hline P248 & $\mathrm{Y} ?$ & 17.92 & $\ldots$ & 2.49 & $\cdots$ & $<10$ & $+2-3$ & 8.0 & 8.8 & 0.0003 & 0.0001 & 29.53 & 32.28 & -2.75 & 1.54 & \\
\hline P230 & $\mathrm{Y} ?$ & 17.57 & $\ldots$ & 2.60 & M3 & 12 & $+2-3$ & 8.2 & 6.0 & 0.00028 & 0.00009 & 29.47 & 32.48 & -3.01 & 1.55 & \\
\hline P257 & $Y ?$ & 17.14 & $\cdots$ & 2.60 & $\ldots$ & 52 & $+3-4$ & 44.0 & 7.6 & 0.0008 & 0.0001 & 29.95 & 32.65 & -2.70 & 1.55 & \\
\hline P270 & $Y ?$ & 17.27 & $\ldots$ & 2.57 & M2.5 & $<10$ & $+2-4$ & 23.6 & 8.4 & 0.0006 & 0.0001 & 29.76 & 32.58 & -2.82 & 1.55 & \\
\hline P283 & $\mathbf{Y} ?$ & 18.25 & $\ldots$ & 2.55 & M3 & $\ldots$ & $-1-1$ & 5.9 & 7.2 & 0.00020 & 0.00008 & 29.32 & 32.18 & -2.86 & 1.55 & \\
\hline P315 & $Y ?$ & 17.85 & $\ldots$ & 2.78 & M3 & $\ldots$ & $+9-2$ & 11.5 & 10.2 & 0.0005 & 0.0001 & 29.74 & 32.45 & -2.71 & 1.58 & \\
\hline P237 & $?$ & 19.36 & $\ldots$ & 3.03 & $\cdots$ & $\ldots$ & $+2-4$ & 10.5 & 5.3 & 0.00029 & 0.00009 & 29.49 & 31.97 & -2.48 & 1.63 & \\
\hline K56 & $\mathbf{N}$ & 7.51 & 0.12 & $\cdots$ & A 0 & $\ldots$ & $+0-1$ & 465.1 & 16.3 & 0.0083 & 0.0004 & 30.94 & 36.03 & -5.09 & -.06 & \\
\hline K60 & $\mathrm{N}$ & 11.0 & $\ldots$ & $\ldots$ & $\mathrm{G} 1$ & $\cdots$ & $+2-2$ & 40.5 & 4.7 & 0.0007 & 0.0001 & 29.86 & 34.39 & -4.53 & 0.60 & \\
\hline P12 & $\mathrm{N}$ & 12.72 & 0.77 & 0.80 & G8V & 70 & $+1-3$ & 627.5 & 6.1 & 0.0042 & 0.0003 & 30.64 & 33.70 & -3.06 & 0.59 & \\
\hline P20 & $\mathbf{N}$ & 12.24 & 0.46 & 0.60 & $\mathrm{~F} 6 \mathrm{~V}$ & $\ldots$ & $+3-4$ & 29.1 & 2.3 & 0.00043 & 0.00009 & 29.66 & 33.91 & -4.25 & 0.28 & \\
\hline P30 & N & 12.68 & 0.61 & 0.65 & G3V & $\ldots$ & $-6-7$ & 24.3 & 18.3 & 0.0016 & 0.0003 & 30.23 & 33.71 & -3.48 & 0.43 & \\
\hline P108 & $\mathbf{N}$ & 13.75 & 0.78 & 0.77 & G8V & $\ldots$ & $+5-3$ & 210.5 & 10.8 & 0.0027 & 0.0002 & 30.45 & 33.29 & -2.84 & 0.60 & \\
\hline
\end{tabular}




\begin{tabular}{|c|c|c|c|c|c|c|c|c|c|c|c|c|c|c|c|c|c|c|c|c|}
\hline Star & Mem & $\begin{array}{c}\text { Cnts } \\
\left(\sec ^{-1}\right)\end{array}$ & $\log L x$ & $\begin{array}{c}\log \\
\left(L_{\mathbf{X}} / L_{\text {bol }}\right)\end{array}$ & $B-V_{0}$ & $\begin{array}{c}\text { Offax } \\
\text { (arcmin) }\end{array}$ & Star & Mem & $\begin{array}{c}\text { Cnts } \\
\left(\sec ^{-1}\right)\end{array}$ & $\log L_{x}$ & $\begin{array}{c}\log \\
\left(L_{x} / L_{\text {bal }}\right)\end{array}$ & $B-V_{0}$ & $\begin{array}{c}\text { Offax } \\
\text { (arcmin) }\end{array}$ & Star & Mem & $\begin{array}{c}\text { Cnts } \\
\left(\sec ^{-1}\right)\end{array}$ & $\log L_{x}$ & $\begin{array}{c}\log \\
\left(L_{x} / L_{\text {bal }}\right)\end{array}$ & B-V。 & $\begin{array}{c}\text { Offax } \\
(\operatorname{arcmin})\end{array}$ \\
\hline K 37 & $\mathbf{Y}$ & 0.00034 & 29.55 & -4.70 & 0.35 & 18.8 & P197 & $?$ & 0.00052 & 29.74 & -2.09 & 1.67 & 14.6 & P282 & $?$ & 0.00022 & 29.37 & -3.30 & 1.10 & 12.4 \\
\hline K 39 & $\mathbf{Y}$ & 0.00039 & 29.61 & -5.47 & 0.12 & 12.7 & P198 & $?$ & 0.00033 & 29.54 & -2.28 & 1.74 & 12.8 & P284 & $?$ & 0.00024 & 29.40 & -2.40 & 1.57 & 13.5 \\
\hline K 43 & $\mathbf{Y}$ & 0.00052 & 29.74 & -5.55 & -0.01 & 6.7 & P199 & $?$ & 0.00029 & 29.49 & -3.52 & 0.83 & 11.5 & P286 & $?$ & 0.00016 & 29.23 & -3.04 & 1.52 & 6.6 \\
\hline K 49 & $\mathbf{Y}$ & 0.00026 & 29.44 & -6.80 & -0.16 & 5.2 & P200 & $?$ & 0.00040 & 29.63 & -2.18 & 1.64 & 13.2 & P287 & $?$ & 0.00031 & 29.51 & -3.54 & 0.98 & 11.8 \\
\hline K 50 & $\mathbf{Y}$ & 0.00047 & 29.70 & -5.51 & 0.08 & 13.0 & P201 & $?$ & 0.00017 & 29.25 & -3.37 & 1.79 & 10.6 & P288 & $?$ & 0.00041 & 29.64 & -2.62 & 1.36 & 13.6 \\
\hline K 57 & $\mathbf{Y}$ & 0.00033 & 329.54 & -4.80 & 0.30 & 10.9 & P203 & $?$ & 0.00032 & 29.53 & -3.40 & 0.97 & 10.6 & P289 & $?$ & 0.00030 & 29.50 & -3.44 & 1.04 & 14.4 \\
\hline K 65 & $\mathbf{Y}$ & 0.00032 & 29.53 & -5.03 & 0.26 & 19.8 & P204 & $?$ & 0.00045 & 29.68 & -2.17 & 1.54 & 17.0 & P291 & $?$ & 0.00064 & 29.83 & -1.84 & 1.63 & 16.7 \\
\hline K 66 & $Y ?$ & 0.00044 & 29.67 & -5.00 & 0.12 & 13.6 & P205 & $?$ & 0.00031 & 29.51 & -2.17 & 1.60 & 12.7 & P292 & $?$ & 0.00017 & 29.25 & -2.82 & 1.42 & 7.9 \\
\hline K $76 A$ & $\mathbf{Y}$ & 0.00068 & 29.86 & -5.90 & -0.07 & 16.5 & P206 & $?$ & 0.00050 & 29.72 & -3.20 & 1.43 & 19.0 & P293 & $?$ & 0.00077 & 29.91 & -3.12 & 1.50 & 18.5 \\
\hline K $76 \mathrm{~B}$ & $\mathbf{Y}$ & 0.00068 & 29.86 & -5.14 & 9.00 & 16.5 & P208 & $?$ & 0.00035 & 29.57 & -3.58 & 1.09 & 11.8 & P295 & $?$ & 0.00031 & 29.51 & -3.52 & 1.01 & 16.4 \\
\hline K 82 & $\mathbf{Y}$ & 0.00019 & 29.30 & -6.68 & -0.12 & 19.8 & P209 & $?$ & 0.00043 & 29.66 & -2.11 & 1.66 & 11.9 & P296 & $?$ & 0.00015 & 29.20 & -3.53 & 1.05 & 7.3 \\
\hline P 19 & $Y$ & 0.00022 & 29.37 & -4.63 & 0.46 & 4.6 & P210 & $?$ & 0.00015 & 29.20 & -2.58 & 1.58 & 9.0 & P297 & $?$ & 0.00027 & 29.45 & -3.99 & 1.39 & 10.5 \\
\hline P 27 & $\mathbf{Y}$ & 0.00095 & 30.00 & -3.73 & 0.59 & 16.2 & P211 & $?$ & 0.00028 & 29.47 & -3.77 & 1.37 & 9.9 & P298 & $?$ & 0.00024 & 29.40 & -2.55 & 1.56 & 11.0 \\
\hline P 39 & $\mathbf{Y}$ & 0.00094 & 30.00 & -3.61 & 0.57 & 15.5 & P212 & $?$ & 0.00060 & 29.80 & -2.07 & 1.64 & 13.9 & P299 & $?$ & 0.00062 & 29.82 & -2.44 & 1.51 & 17.8 \\
\hline P 75 & $\mathrm{Y}$ & 0.00069 & 29.86 & -3.48 & 0.71 & 14.1 & P213 & $?$ & 0.00034 & 29.55 & -3.42 & 0.96 & 14.2 & P300 & $?$ & 0.00012 & 29.10 & -2.52 & 1.64 & 7.9 \\
\hline P 77 & $Y ?$ & 0.00060 & 29.80 & -3.16 & 1.07 & 14.7 & P214 & $?$ & 0.00045 & 29.68 & -3.11 & 1.10 & 13.8 & P301 & $?$ & 0.00036 & 29.58 & -3.06 & 1.43 & 14.5 \\
\hline P 83 & $\mathbf{Y} ?$ & 0.00032 & 29.53 & -3.31 & 1.11 & 13.8 & P215 & $?$ & 0.00034 & 29.55 & -3.53 & 1.03 & 11.4 & P303 & $?$ & 0.00015 & 29.20 & -3.00 & 1.50 & 9.1 \\
\hline P 94 & $\mathbf{Y}$ & 0.00010 & 29.02 & -4.12 & 0.83 & 3.1 & P216 & $?$ & 0.00038 & 29.60 & -2.37 & 1.54 & 12.5 & P304 & $?$ & 0.00051 & 29.73 & -2.07 & 1.54 & 19.0 \\
\hline Р 99 & $\mathbf{Y} ?$ & 0.00025 & 29.42 & -3.49 & 1.17 & 12.1 & P217 & $?$ & 0.00077 & 29.91 & -2.24 & 1.54 & 19.4 & P306 & $?$ & 0.00029 & 29.49 & -3.53 & 0.96 & 10.9 \\
\hline P120 & $Y ?$ & 0.00031 & 29.51 & -3.68 & 1.19 & 18.1 & P218 & $?$ & 0.00028 & 29.47 & -3.02 & 1.46 & 10.2 & P308 & $?$ & 0.00050 & 29.72 & -3.50 & 1.15 & 18.7 \\
\hline P121 & $Y ?$ & 0.00033 & 29.54 & -3.56 & 1.19 & 18.8 & P220 & $?$ & 0.00021 & 29.35 & -2.26 & 1.62 & 10.5 & P310 & $?$ & 0.00054 & 29.76 & -3.11 & 1.15 & 14.6 \\
\hline P126 & $Y ?$ & 0.00072 & 29.88 & -3.16 & 1.26 & 17.8 & P222 & $?$ & 0.00066 & 29.84 & -3.15 & 1.04 & 18.7 & P311 & $?$ & 0.00025 & 29.42 & -2.52 & 1.54 & 11.3 \\
\hline P243 & $Y ?$ & 0.00017 & 29.25 & -3.20 & 1.57 & 9.3 & P223 & $?$ & 0.00020 & 29.32 & -2.82 & 1.38 & 7.6 & P312 & $?$ & 0.00036 & 29.58 & -2.36 & 1.75 & 15.0 \\
\hline P265 & $Y ?$ & 0.00019 & 29.30 & -3.24 & 1.50 & 5.6 & P224 & $?$ & 0.00037 & 29.59 & -2.48 & 1.63 & 15.2 & P313 & $?$ & 0.00035 & 29.57 & -2.24 & 1.64 & 13.1 \\
\hline P283 & $Y ?$ & 0.00031 & 29.51 & -2.72 & 1.55 & 7.2 & P225 & $?$ & 0.00044 & 29.67 & -2.69 & 1.28 & 15.6 & P314 & $?$ & 0.00039 & 29.61 & -2.74 & 1.35 & 16.4 \\
\hline P305 & $Y ?$ & 0.00039 & 29.61 & -2.74 & 1.56 & 17.3 & P227 & $?$ & 0.00040 & 29.63 & -2.63 & 1.52 & 16.9 & P316 & $?$ & 0.00041 & 29.64 & -3.13 & 1.38 & 13.6 \\
\hline P309 & Y? & 0.00070 & 29.87 & -2.69 & 1.41 & 11.9 & P228 & $?$ & 0.00017 & 29.25 & -2.94 & 1.49 & 6.6 & P317 & $?$ & 0.00041 & 29.64 & -3.17 & 1.38 & 13.6 \\
\hline P335 & $\mathrm{Y} ?$ & 0.00031 & 29.51 & -2.61 & 1.63 & 13.1 & P231 & $?$ & 0.00018 & 29.28 & -2.46 & 1.71 & 6.9 & P320 & $?$ & 0.00035 & 29.57 & -2.45 & 1.52 & 14.1 \\
\hline P336 & $Y ?$ & 0.00033 & 29.54 & -2.93 & 1.50 & 14.9 & P233 & $?$ & 0.00033 & 29.54 & -2.54 & 1.71 & 16.0 & P321 & $?$ & 0.00033 & 29.54 & -1.75 & 1.73 & 11.5 \\
\hline P343 & $\mathbf{Y} ?$ & 0.00040 & 29.63 & -2.76 & 1.56 & 15.5 & P234 & $?$ & 0.00012 & 29.10 & -3.18 & 1.33 & 5.8 & P322 & $?$ & 0.00021 & 29.35 & -3.48 & 1.09 & 11.4 \\
\hline P348 & $Y ?$ & 0.00053 & 29.75 & -2.43 & 1.60 & 17.2 & P235 & $?$ & 0.00027 & 29.45 & -2.09 & 1.73 & 6.6 & P323 & $?$ & 0.00023 & 29.39 & -2.77 & 1.43 & 12.2 \\
\hline P354 & $Y ?$ & 0.00057 & 29.78 & -2.56 & 1.56 & 17.4 & P238 & $?$ & 0.00030 & 29.50 & -2.36 & 1.85 & 19.4 & P324 & $?$ & 0.00055 & 29.76 & -3.42 & 1.28 & 19.9 \\
\hline P373 & $Y ?$ & 0.00070 & 29.87 & -2.16 & 1.70 & 18.4 & P240 & $?$ & 0.00050 & 29.72 & -2.10 & 1.93 & 18.7 & P326 & $?$ & 0.00046 & 29.69 & -3.24 & 0.97 & 15.0 \\
\hline K 38 & $?$ & 0.00063 & 29.82 & -4.68 & 0.35 & 17.9 & P241 & $?$ & 0.00046 & 29.69 & -2.02 & 1.64 & 11.9 & P328 & $?$ & 0.00027 & 29.45 & -3.10 & 1.01 & 14.0 \\
\hline P 2 & $?$ & 0.00070 & 29.87 & -4.27 & 0.25 & 15.9 & P244 & $?$ & 0.00021 & 29.35 & -2.57 & 1.61 & 3.1 & P329 & $?$ & 0.00028 & 29.47 & -2.77 & 1.42 & 14.9 \\
\hline P 15 & ? & 0.00014 & 29.17 & -4.32 & 0.48 & 5.0 & P245 & $?$ & 0.00007 & 28.87 & -3.16 & 1.44 & 3.2 & P331 & $?$ & 0.00017 & 29.25 & -3.58 & 0.96 & 12.9 \\
\hline P 59 & $?$ & 0.00024 & 29.40 & -3.91 & 1.09 & 16.2 & P246 & $?$ & 0.00012 & 29.10 & -2.63 & 1.69 & 3.0 & P332 & $?$ & 0.00033 & 29.54 & -3.51 & 0.91 & 16.5 \\
\hline P 63 & $?$ & 0.00027 & 29.45 & -4.24 & 0.89 & 10.7 & P250 & $?$ & 0.00018 & 29.28 & -3.71 & 1.08 & 10.8 & P333 & $?$ & 0.00026 & 29.44 & -2.40 & 1.73 & 13.7 \\
\hline P 64 & $?$ & 0.00034 & 29.55 & -3.55 & 0.77 & 15.5 & P252 & $?$ & 0.00012 & 29.10 & -3.98 & 1.01 & 5.6 & P337 & $?$ & 0.00029 & 29.49 & -2.40 & 1.60 & 13.6 \\
\hline P 65 & $?$ & 0.00036 & 29.58 & -3.86 & 1.04 & 13.8 & P253 & $?$ & 0.00017 & 29.25 & -2.68 & 1.49 & 6.5 & P338 & $?$ & 0.00045 & 29.68 & -2.09 & 1.61 & 14.0 \\
\hline$P_{67}$ & $?$ & 0.00033 & 29.54 & -3.30 & 0.96 & 13.3 & P254 & $?$ & 0.00057 & 29.78 & -3.13 & 1.20 & 14.6 & P339 & $?$ & 0.00063 & 29.82 & -2.99 & 0.97 & 17.1 \\
\hline P 78 & $?$ & 0.00033 & 29.54 & -3.64 & 0.63 & 13.5 & P255 & $?$ & 0.00010 & 29.02 & -3.09 & 1.44 & 1.6 & P340 & $?$ & 0.00043 & 29.66 & -2.23 & 1.70 & 19.2 \\
\hline P 93 & $?$ & 0.00009 & 28.98 & -4.49 & 1.07 & 3.6 & P256 & $?$ & 0.00024 & 29.40 & -2.83 & 1.39 & 10.1 & P341 & $?$ & 0.00019 & 29.30 & -2.47 & 1.61 & 14.4 \\
\hline P101 & $?$ & 0.00029 & 29.49 & -3.76 & 1.33 & 12.0 & P258 & $?$ & 0.00012 & 29.10 & -2.83 & 1.51 & 6.9 & P342 & $?$ & 0.00057 & 29.78 & -2.02 & 1.65 & 17.5 \\
\hline P104 & $?$ & 0.00041 & 29.64 & -3.67 & 1.20 & 14.9 & P259 & $?$ & 0.00058 & 29.79 & -2.06 & 1.63 & 14.5 & P344 & $?$ & 0.00023 & 29.39 & -2.45 & 1.75 & 17.9 \\
\hline P114 & $?$ & 0.00030 & 29.50 & -3.96 & 1.04 & 12.2 & P260 & $?$ & 0.00060 & 29.80 & -2.48 & 1.48 & 16.0 & P346 & $?$ & 0.00052 & 29.74 & -2.98 & 1.04 & 15.4 \\
\hline P119 & $?$ & 0.00037 & 29.59 & -3.77 & 1.14 & 19.2 & P262 & $?$ & 0.00066 & 29.84 & -1.88 & 1.63 & 17.6 & P347 & $?$ & 0.00047 & 29.70 & -2.90 & 1.39 & 15.2 \\
\hline P122 & $?$ & 0.00040 & 29.63 & -3.18 & 0.95 & 17.0 & P263 & $?$ & 0.00021 & 29.35 & -3.66 & 0.96 & 9.0 & P349 & $?$ & 0.00031 & 29.51 & -3.49 & 0.93 & 19.2 \\
\hline P184 & $?$ & 0.00036 & 29.58 & -3.06 & 1.58 & 17.2 & P264 & ? & 0.00011 & 29.06 & -2.56 & 1.64 & 2.2 & P350 & $?$ & 0.00035 & 29.57 & -2.35 & 1.73 & 16.8 \\
\hline P185 & $?$ & 0.00041 & 29.64 & -3.55 & 1.12 & 17.1 & P267 & $?$ & 0.00035 & 29.57 & -3.35 & 0.86 & 12.9 & P352 & $?$ & 0.00041 & 29.64 & -3.35 & 0.90 & 16.1 \\
\hline P186 & $?$ & 0.00023 & 29.39 & -2.04 & 1.66 & 13.4 & P268 & $?$ & 0.00014 & 29.17 & -3.31 & 1.28 & 8.4 & P359 & $?$ & 0.00034 & 29.55 & -2.36 & 1.60 & 16.7 \\
\hline P187 & $?$ & 0.00090 & 29.98 & -2.06 & 1.61 & 18.3 & P269 & $?$ & 0.00028 & 29.47 & -2.72 & 1.62 & 12.3 & P360 & $?$ & 0.00049 & 29.71 & -2.36 & 1.54 & 19.4 \\
\hline P188 & $?$ & 0.00045 & 29.68 & -2.35 & 1.48 & 17.3 & P271 & ? & 0.00008 & 28.93 & -3.85 & 1.28 & 5.8 & P362 & $?$ & 0.00063 & 29.82 & -2.40 & 1.36 & 16.7 \\
\hline P189 & $?$ & 0.00031 & 29.51 & -2.34 & 1.74 & 12.9 & P272 & $?$ & 0.00024 & 29.40 & -2.38 & 1.60 & 14.7 & P364 & $?$ & 0.00070 & 29.87 & -2.17 & 1.47 & 18.4 \\
\hline P190 & $?$ & 0.00055 & 29.76 & -3.06 & 1.06 & 12.3 & P273 & $?$ & 0.00029 & 29.49 & -3.17 & 1.42 & 14.4 & P366 & $?$ & 0.00082 & 29.94 & -2.71 & 1.05 & 18.2 \\
\hline P191 & $?$ & 0.00039 & 29.61 & -2.50 & 1.58 & 18.8 & P274 & $?$ & 0.00027 & 29.45 & -2.17 & 1.73 & 8.2 & P367 & $?$ & 0.00065 & 29.84 & -1.44 & 1.70 & 19.0 \\
\hline P192 & $?$ & 0.00056 & 29.77 & -2.17 & 1.58 & 12.2 & P275 & $?$ & 0.00009 & 28.98 & -3.15 & 1.47 & 4.8 & P369 & $?$ & 0.00080 & 29.93 & -1.89 & 1.68 & 18.1 \\
\hline P193 & $?$ & 0.00054 & 29.76 & -2.97 & 1.35 & 16.7 & P276 & $?$ & 0.00027 & 29.45 & -3.46 & 0.97 & 13.1 & P370 & $?$ & 0.00040 & 29.63 & -2.60 & 1.50 & 19.7 \\
\hline P194 & $?$ & 0.00023 & 29.39 & -3.47 & 1.30 & 12.0 & P277 & $?$ & 0.00009 & 28.98 & -2.95 & 1.51 & 4.7 & P372 & $?$ & 0.00075 & 29.90 & -1.82 & 1.67 & 18.1 \\
\hline P195 & ? & 0.00035 & 29.57 & -2.54 & 1.39 & 16.4 & P278 & $?$ & 0.00008 & 28.93 & -3.34 & 1.39 & 4.7 & & & & & & & \\
\hline P196 & $?$ & 0.00045 & 29.68 & -3.13 & 0.97 & 12.1 & P281 & $?$ & 0.00044 & 29.67 & -2.86 & 1.36 & 12.9 & & & & & & & \\
\hline
\end{tabular}


TABLE 8. Bright Cluster Members

\begin{tabular}{llcc}
\hline \hline Kopff $^{\mathrm{a}}$ & Spt $^{\mathrm{b}}$ & X-ray Activity & $\log \mathrm{L}$ X \\
& & & \\
\hline 62 & B3IV & detected & 29.61 \\
73 & B5IV & detected & 30.53 \\
64 & B5IV & detected & 29.60 \\
105 & B6V SB2 & beyond field & $\ldots$ \\
58 & B6V & detected & 30.64 \\
49 & B6Vp CII SB1 & detected & 29.28 \\
72 & B7V SB1 & beyond field & $\ldots$ \\
82 & B6Vp CII & upper limit & $<29.30$ \\
$23 \mathrm{~A}$ & B8V & beyond field & $\ldots$ \\
$76 \mathrm{~A}$ & B8.5Vp Hg-Mn & upper limit & $<29.86$ \\
32 & B8V shell & beyond field & $\ldots$ \\
22 & B8.5V & beyond field & $\ldots$ \\
$23 \mathrm{~B}$ & B9.5V & beyond field & $\ldots$ \\
81 & B8V & beyond field & $\ldots$ \\
43 & A1V & detected & 29.67 \\
50 & A1+A2V SB2 & upper limit & $<29.70$ \\
102 & A2V & beyond field & $\ldots$ \\
39 & Am (classical) & upper limit & $<29.61$ \\
67 A & A2V VB & (detected) & 30.12 \\
& & & \\
\hline
\end{tabular}

a Kopff (1943)

b Abt \& Levato (1975) 
TABLE 9. Saturation Colors for Other Clusters

\begin{tabular}{lcll}
\hline \hline Cluster & $\begin{array}{c}\text { Age } \\
(\mathrm{Myr})\end{array}$ & B-V。 & Reference \\
\hline IC2602 $\ldots \ldots \ldots \ldots$ & $\sim 30$ & $\sim 0.65$ & Randich et al. 1995 \\
$\alpha$ Per $\ldots \ldots \ldots \ldots$ & $\sim 50$ & $\sim 0.6$ & Prosser et al. 1996 \\
Pleiades $\ldots \ldots \ldots$ & $\sim 70$ & $\sim 0.75$ & Fig. 4 \\
NGC6475 ...... & $\sim 220$ & $\sim 1.0$ & Prosser et al. 1995 \\
Praesepe $/$ Hyades & $\sim 600-800$ & $\sim 1.4$ & Randich \& Schmitt 1995 \\
& & & Stern et al. 1995 \\
\hline
\end{tabular}


TABLE 10. Candidate Variable X-ray Sources

\begin{tabular}{|c|c|c|c|c|c|c|c|}
\hline \multirow[b]{2}{*}{ Name } & \multirow[b]{2}{*}{ Mem } & \multicolumn{2}{|c|}{ IC4665-A } & \multicolumn{2}{|c|}{ IC4665-B } & \multirow[b]{2}{*}{$M L(A)$} & \multirow[b]{2}{*}{$\mathrm{ML}(\mathrm{B})$} \\
\hline & & $\begin{array}{l}\text { Rate } \\
\left(\mathrm{s}^{-1}\right)\end{array}$ & $\begin{array}{l}\text { Error } \\
\left(\mathrm{s}^{-1}\right)\end{array}$ & $\begin{array}{l}\text { Rate } \\
\left(\mathrm{s}^{-1}\right)\end{array}$ & $\begin{array}{l}\text { Error } \\
\left(s^{-1}\right)\end{array}$ & & \\
\hline K56 & $\mathrm{N}$ & 0.0070 & 0.0005 & 0.0097 & 0.0007 & 200.0 & 248.2 \\
\hline K43 & $\mathbf{Y}$ & 0.0007 & 0.0002 & $<0.00003$ & $\ldots .$. & 5.9 & 0.1 \\
\hline P230 & Y? & 0.0003 & 0.0001 & $<0.0003$ & $\ldots \ldots$ & 5.2 & 3.0 \\
\hline P232 & $?$ & 0.0013 & 0.0002 & $<0.0003$ & $\ldots .$. & 41.3 & 1.7 \\
\hline P237 & $?$ & 0.0005 & 0.0001 & $<0.0001$ & $\ldots$. & 10.7 & 1.1 \\
\hline P248 & Y? & $<0.0002$ & 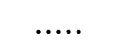 & 0.0006 & 0.0002 & 1.2 & 8.4 \\
\hline R6 & $\mathrm{N}$ & 0.0005 & 0.0002 & $\ldots \ldots$ & $\ldots .$. & 10.5 & 0.0 \\
\hline R12 & $\mathrm{N}$ & $<0.0002$ & $\ldots$. & 0.0007 & 0.0002 & 2.6 & 10.9 \\
\hline
\end{tabular}


TABLE 11. Additional X-ray Sources

\begin{tabular}{|c|c|c|c|c|c|c|c|}
\hline ID & RA (2000) & DEC & ML & $\begin{array}{c}\text { Offax } \\
(\operatorname{arcmin})\end{array}$ & $\begin{array}{c}\text { Cnts } \\
\left(\sec ^{-1}\right)\end{array}$ & $\begin{array}{c}\text { Err } \\
\left(\sec ^{-1}\right)\end{array}$ & $\log L_{X}$ \\
\hline R1 & 174501.7 & $\begin{array}{ll}53927 \\
\end{array}$ & 11.9 & 10.4 & 0.0005 & 0.0001 & 29.75 \\
\hline $\mathrm{R} 2$ & 174505.7 & 54739 & 9.4 & 13.1 & 0.0007 & 0.0002 & 29.84 \\
\hline R3 & 174513.9 & 53753 & 15.3 & 7.3 & 0.0005 & 0.0001 & 29.72 \\
\hline R4 & 174514.4 & 53043 & 11.1 & 10.5 & 0.0005 & 0.0001 & 29.72 \\
\hline R5 & 174518.5 & 54500 & 7.9 & 9.0 & 0.0003 & 0.0001 & 29.55 \\
\hline R6 & 174524.4 & 53306 & 7.1 & 7.1 & 0.0004 & 0.0001 & 29.57 \\
\hline R7 & 174543.2 & $\begin{array}{lll}55516 & \end{array}$ & 12.2 & 16.9 & 0.0011 & 0.0002 & 30.05 \\
\hline R8 & 174546.7 & 53726 & 18.8 & 1.3 & 0.0005 & 0.0001 & 29.71 \\
\hline R9 & 174548.5 & 54448 & 27.7 & 6.5 & 0.0005 & 0.0001 & 29.70 \\
\hline R10 & 174555.2 & 53755 & 18.2 & 3.0 & 0.0003 & 0.0001 & 29.50 \\
\hline R11 & 174557.3 & 54656 & 5.7 & 9.2 & 0.0003 & 0.0001 & 29.47 \\
\hline R12 & 174603.8 & 53938 & 13.8 & 5.3 & 0.0004 & 0.0001 & 29.65 \\
\hline R13 & 174606.3 & 53232 & 8.7 & 8.2 & 0.0003 & 0.0001 & 29.45 \\
\hline R14 & 174607.6 & 55354 & 123.5 & 16.6 & 0.0041 & 0.0003 & 30.63 \\
\hline R15 & $1746 \quad 37.2$ & 54334 & 12.7 & 14.4 & 0.0009 & 0.0002 & 29.96 \\
\hline
\end{tabular}


TABLE 12. Stars Associated with X-ray Sources

\begin{tabular}{|c|c|c|c|c|c|c|c|c|c|}
\hline Star & V & $B-V$ & $V-I_{K}$ & Mem & Star & V & $B-V$ & $V-I_{K}$ & Mem \\
\hline R1A & 14.37 & 0.86 & 0.79 & $N$ & R10B & 18.80 & $\ldots$ & 0.80 & $\mathrm{~N}$ \\
\hline R3A & 17.23 & 1.08 & 1.00 & $\mathrm{~N}$ & $\mathrm{R} 10 \mathrm{C}$ & 16.89 & $\ldots$ & 0.63 & $N$ \\
\hline R3B & 18.27 & 0.92 & 1.13 & $\mathrm{~N}$ & R10D & 17.58 & $\ldots$ & 0.95 & $N$ \\
\hline $\mathrm{R} 3 \mathrm{C}$ & 17.98 & 0.87 & 0.62 & $\mathrm{~N}$ & R10E & 14.03 & & 0.36 & $\mathrm{~N}$ \\
\hline R3D & 17.31 & 0.76 & 0.47 & $\mathrm{~N}$ & R11A & 16.81 & 0.80 & 0.69 & $\mathrm{~N}$ \\
\hline R3E & 13.60 & 0.84 & 0.74 & $?$ & R11B & 17.94 & 0.81 & 0.68 & $\mathrm{~N}$ \\
\hline R3F & 15.21 & 0.97 & 0.84 & $\mathbf{N}$ & R11C & 18.78 & 1.05 & 1.07 & $\mathbf{N}$ \\
\hline R3G & 17.75 & 0.74 & 1.01 & $\mathrm{~N}$ & R11D & 18.40 & 1.28 & 1.17 & $\mathrm{~N}$ \\
\hline R3H & 19.10 & 1.57 & 1.83 & $\mathbf{N}$ & R11E & 17.77 & 1.01 & 0.87 & $\mathrm{~N}$ \\
\hline R4A & 15.05 & 1.17 & 1.06 & $?$ & R11F & 19.55 & $\ldots$ & 1.29 & $\mathrm{~N}$ \\
\hline R4B & 15.83 & 0.90 & 0.69 & $\mathrm{~N}$ & R11G & 19.28 & $\ldots$ & 1.05 & $\mathrm{~N}$ \\
\hline $\mathrm{R} 4 \mathrm{C}$ & 15.45 & 0.89 & 0.82 & $\mathbf{N}$ & R12A: & 14.91 & $\ldots$ & 0.71 & $\mathrm{~N}$ \\
\hline R4D & 16.66 & 0.94 & 0.85 & $\mathbf{N}$ & R12B: & 16.53 & $\ldots$ & 0.61 & $\mathrm{~N}$ \\
\hline R4E: & 19.17 & & 1.01 & $\mathrm{~N}$ & R12C & 16.78 & $\ldots$ & 1.42 & $\mathrm{~N}$ \\
\hline R6A & 15.80 & 1.19 & 1.02 & $\mathbf{N}$ & R12D & 18.33 & $\ldots$ & 0.68 & $\mathrm{~N}$ \\
\hline $\mathrm{R} 6 \mathrm{~B}$ & 16.97 & 0.70 & 0.76 & $\mathbf{N}$ & R12E & 18.40 & $\ldots$ & 0.87 & $\mathrm{~N}$ \\
\hline $\mathrm{R} 6 \mathrm{C}$ & 15.79 & 0.93 & 0.85 & $\mathbf{N}$ & R12F & 18.87 & $\ldots$ & 2.37 & N? \\
\hline R7A: & 14.26 & 1.16 & 0.97 & $Y ?$ & R12G & 17.47 & $\ldots$ & 0.51 & $\mathbf{N}$ \\
\hline R7B & 18.98 & & 1.27 & $\mathrm{~N}$ & R13A & 17.21 & $\ldots$ & 1.13 & $\mathrm{~N}$ \\
\hline R7C & 15.33 & 1.01 & 0.91 & $\mathrm{~N}$ & R13B & 19.81 & $\ldots$ & 1.53 & $N$ \\
\hline R8A & 19.18 & $\ldots$ & 0.36 & $\mathrm{~N}$ & R13C & 19.49 & $\cdots$ & 0.81 & $N$ \\
\hline R8B & 20.12 & $\cdots$ & 0.85 & $\mathrm{~N}$ & R13D & 18.05 & $\ldots$ & 0.98 & $N$ \\
\hline R8C & 19.55 & $\ldots$ & 0.93 & $\mathrm{~N}$ & R13E & 18.76 & $\ldots$ & 1.12 & $\mathrm{~N}$ \\
\hline R8D & 20.50 & $\ldots$ & 1.97 & $\mathrm{~N}$ & R13F & 17.44 & $\ldots$ & 0.87 & $\mathrm{~N}$ \\
\hline R8E & 15.57 & $\ldots$ & 0.96 & $\mathbf{N}$ & R13G & 18.18 & $\cdots$ & 1.40 & $\mathrm{~N}$ \\
\hline R8F & 16.78 & & 0.65 & $\mathrm{~N}$ & R13H & 16.21 & $\ldots$ & 0.78 & $\mathrm{~N}$ \\
\hline R9A & 14.80 & 0.92 & 0.81 & $\mathbf{N}$ & R13I & 18.30 & $\ldots$ & 1.77 & $N$ \\
\hline R9B & 15.61 & 0.59 & 0.54 & $\mathbf{N}$ & R13J & 16.80 & $\ldots$ & 0.68 & $N$ \\
\hline R9C & 18.78 & 0.55 & 0.54 & $\mathrm{~N}$ & $\mathrm{R} 14^{a}$ & 13.60 & 1.36 & 1.62 & $\mathrm{~N}$ \\
\hline R9D & 13.32 & $\cdots$ & 0.67 & $?$ & R15A & 19.36 & $\ldots$ & 2.46 & $\mathrm{~N} ?$ \\
\hline R10A & 16.92 & $\ldots$ & 0.13 & $\mathrm{~N}$ & R15B & 18.72 & $\ldots$ & 1.07 & $\mathrm{~N}$ \\
\hline
\end{tabular}

a companion to $R 14: I_{K} \simeq 16, V \geq 20-21$, sep $\approx 3^{\prime \prime}$ south 

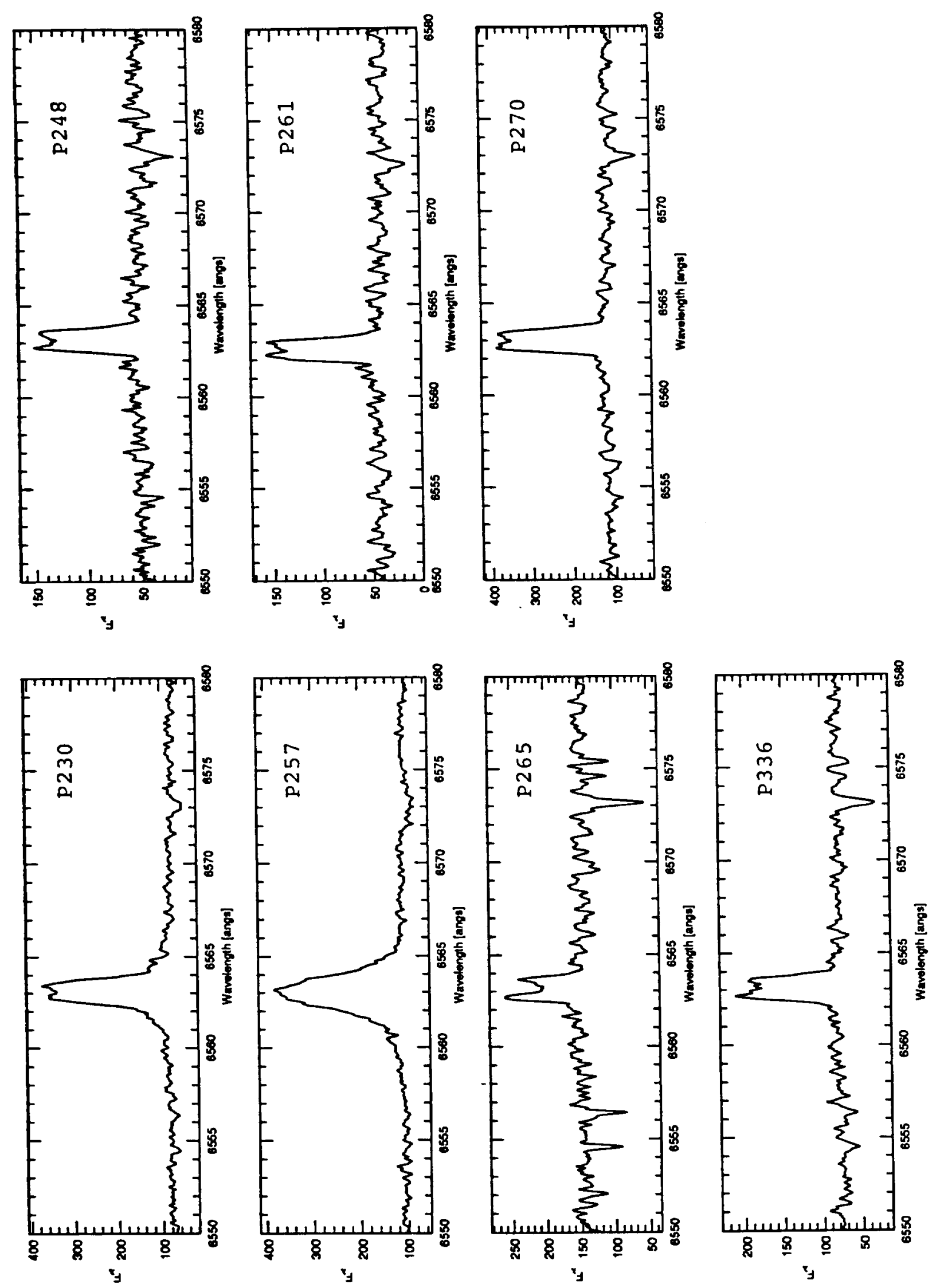


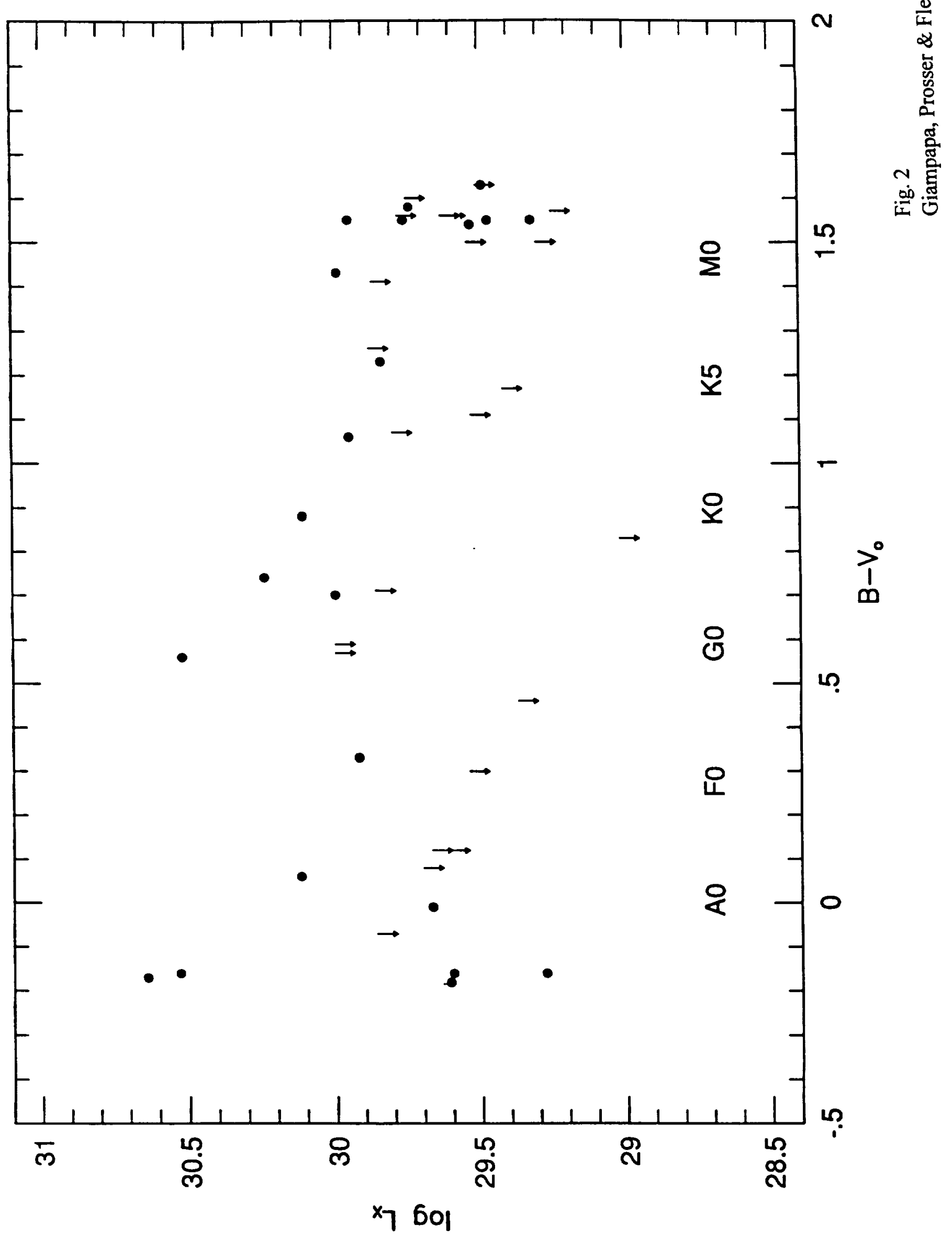




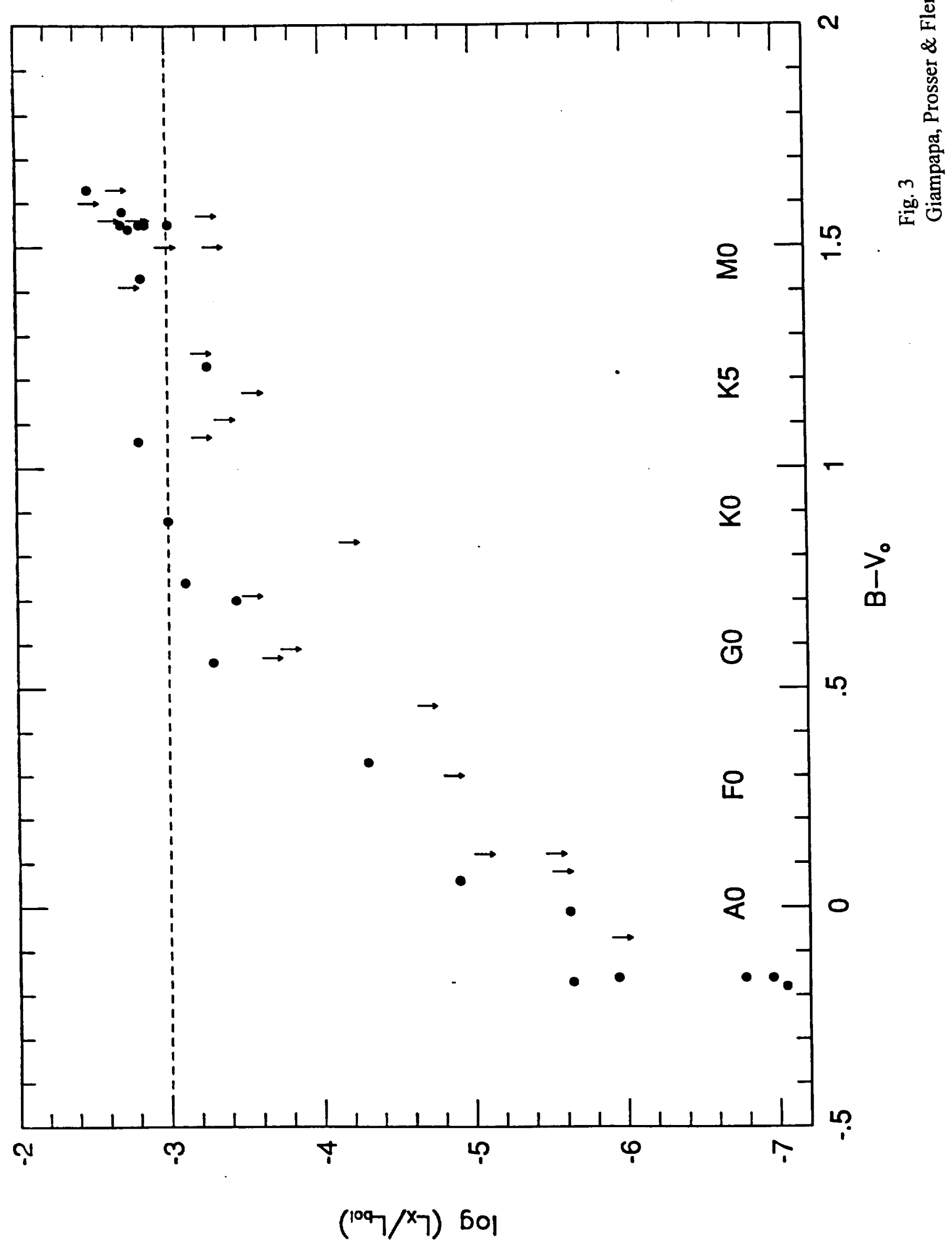




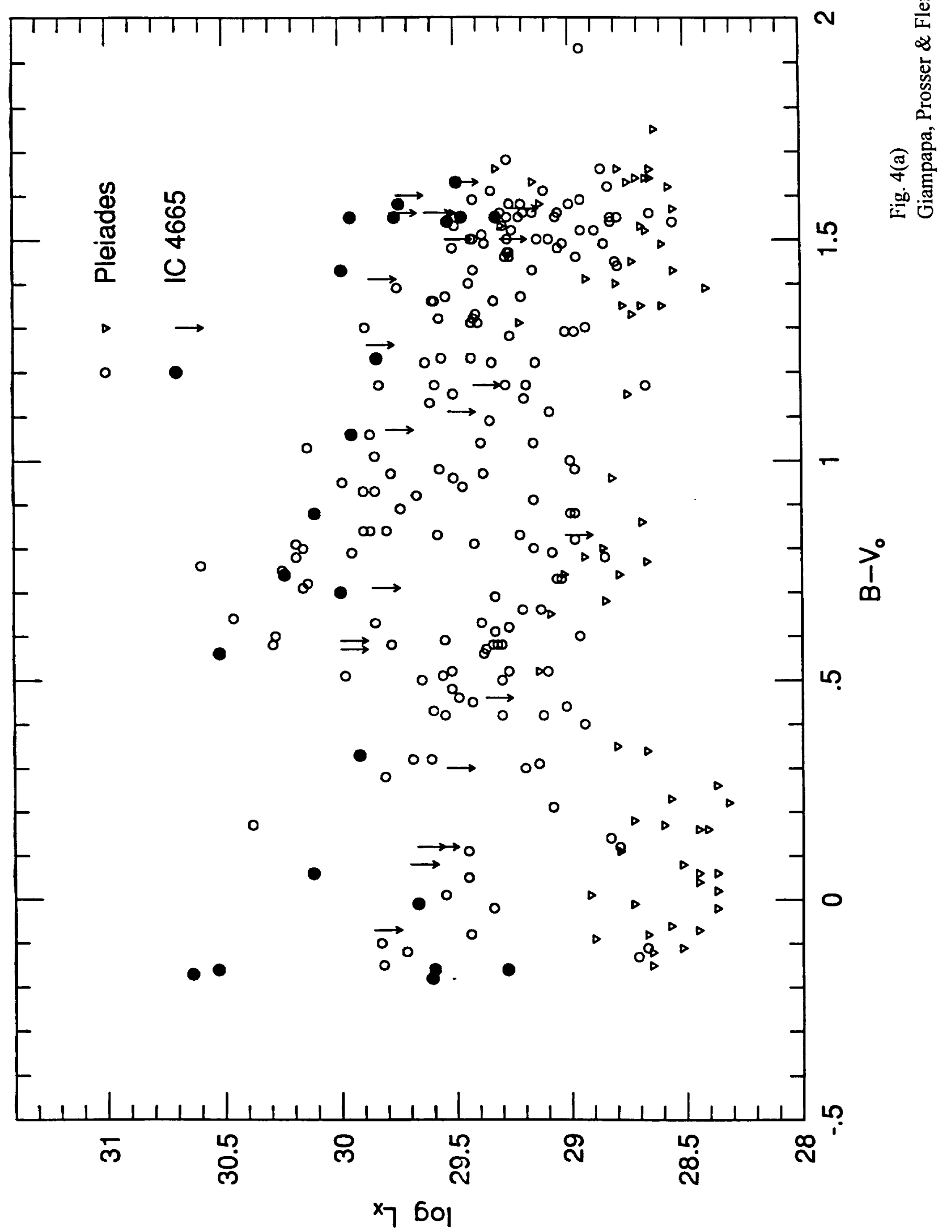




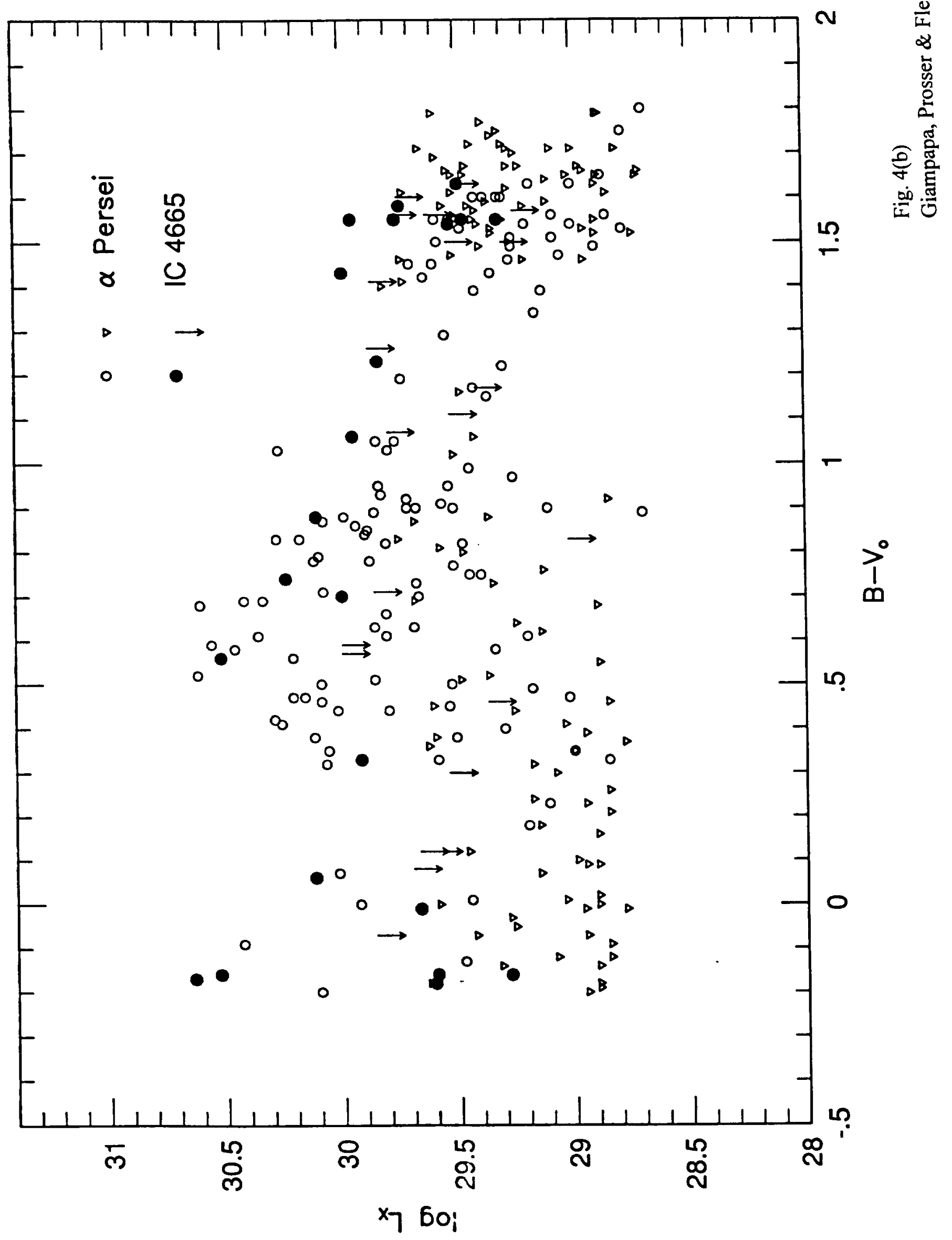




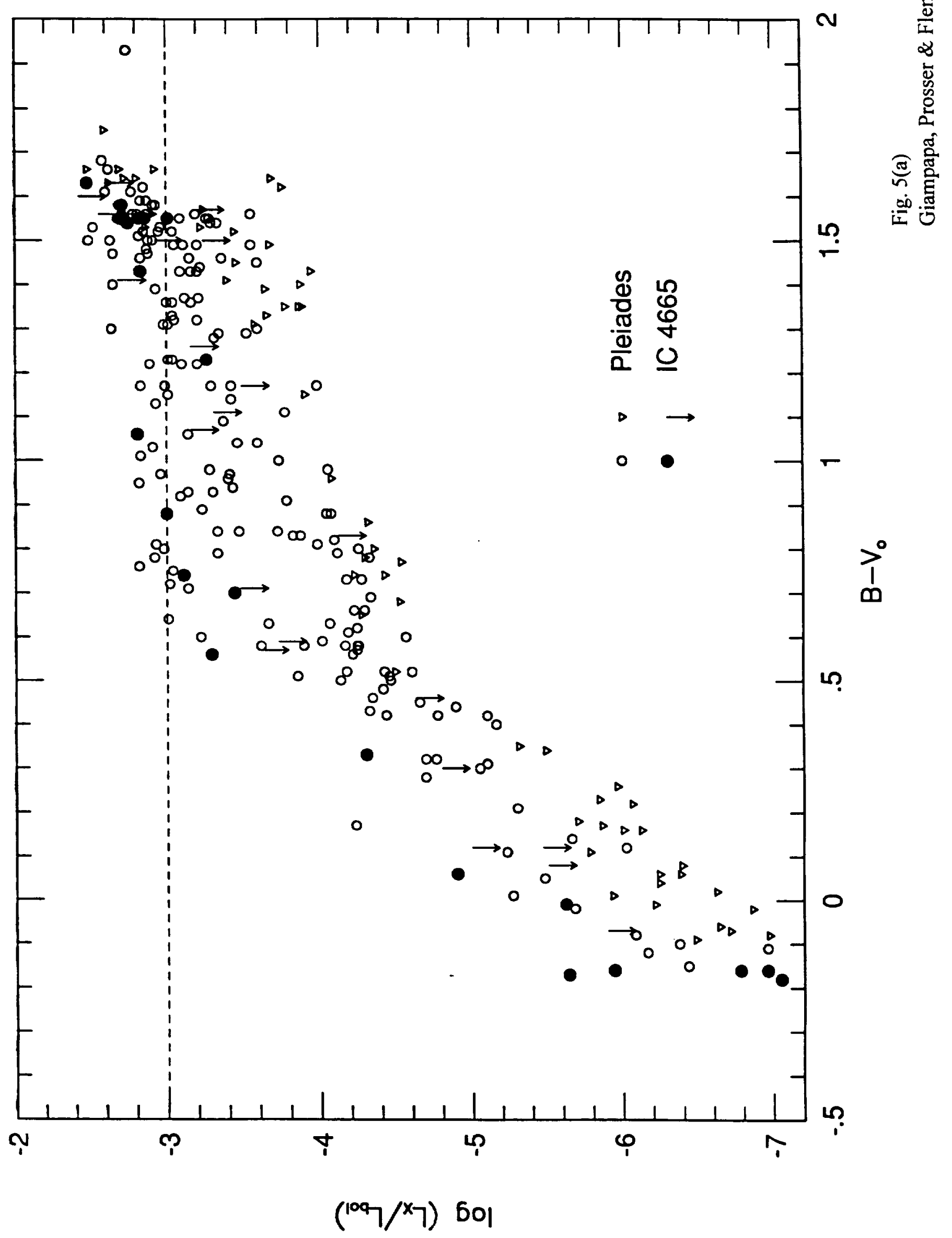




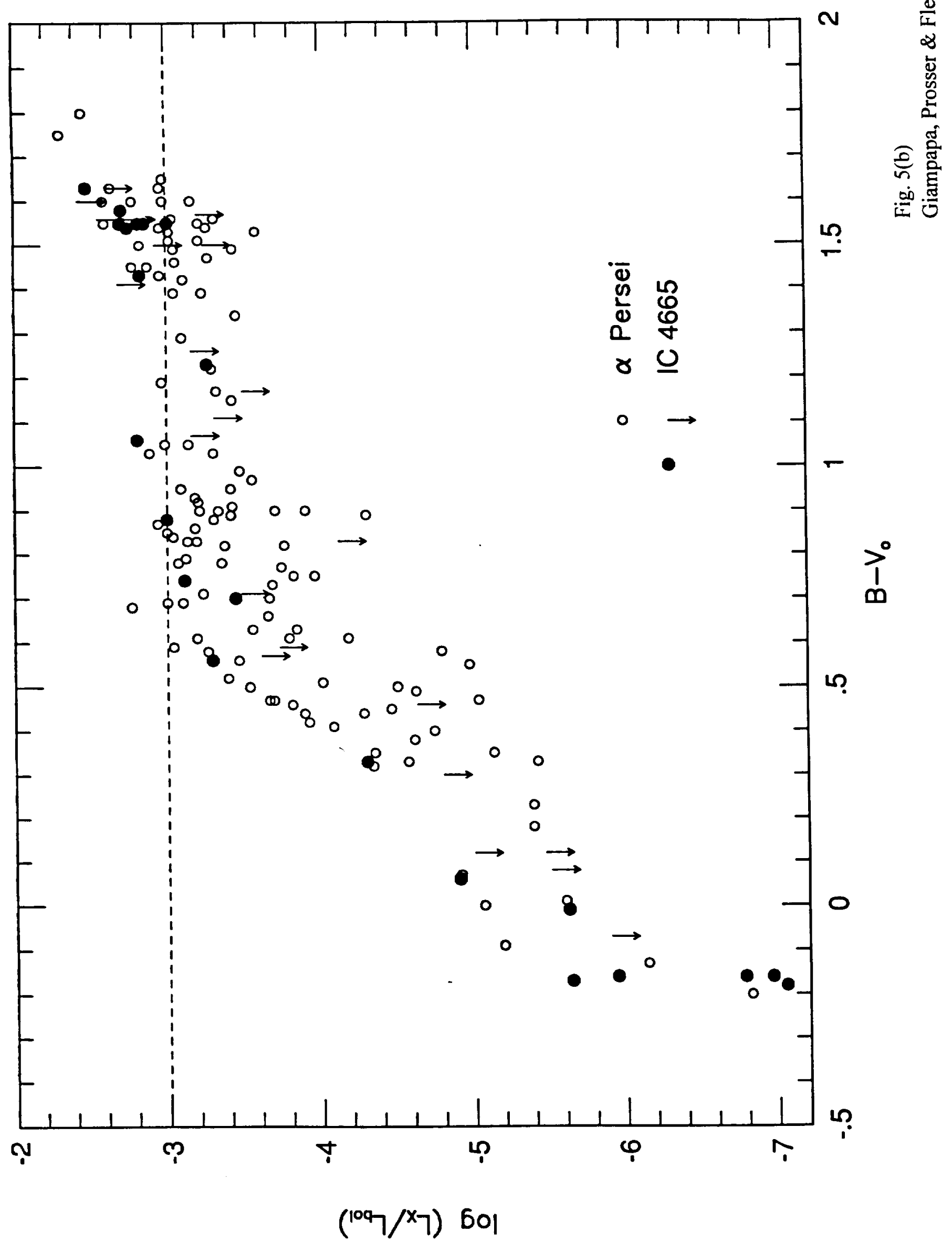




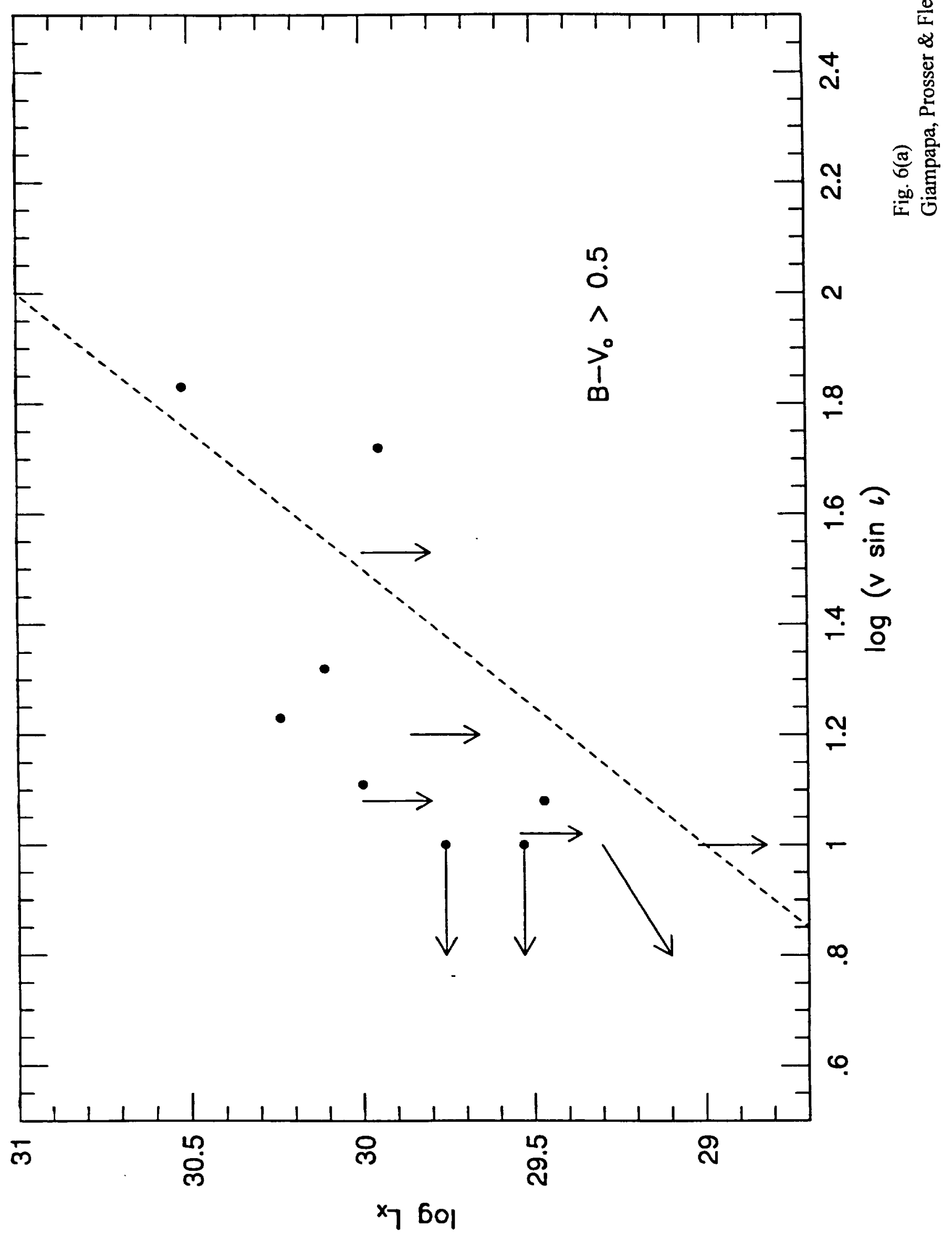




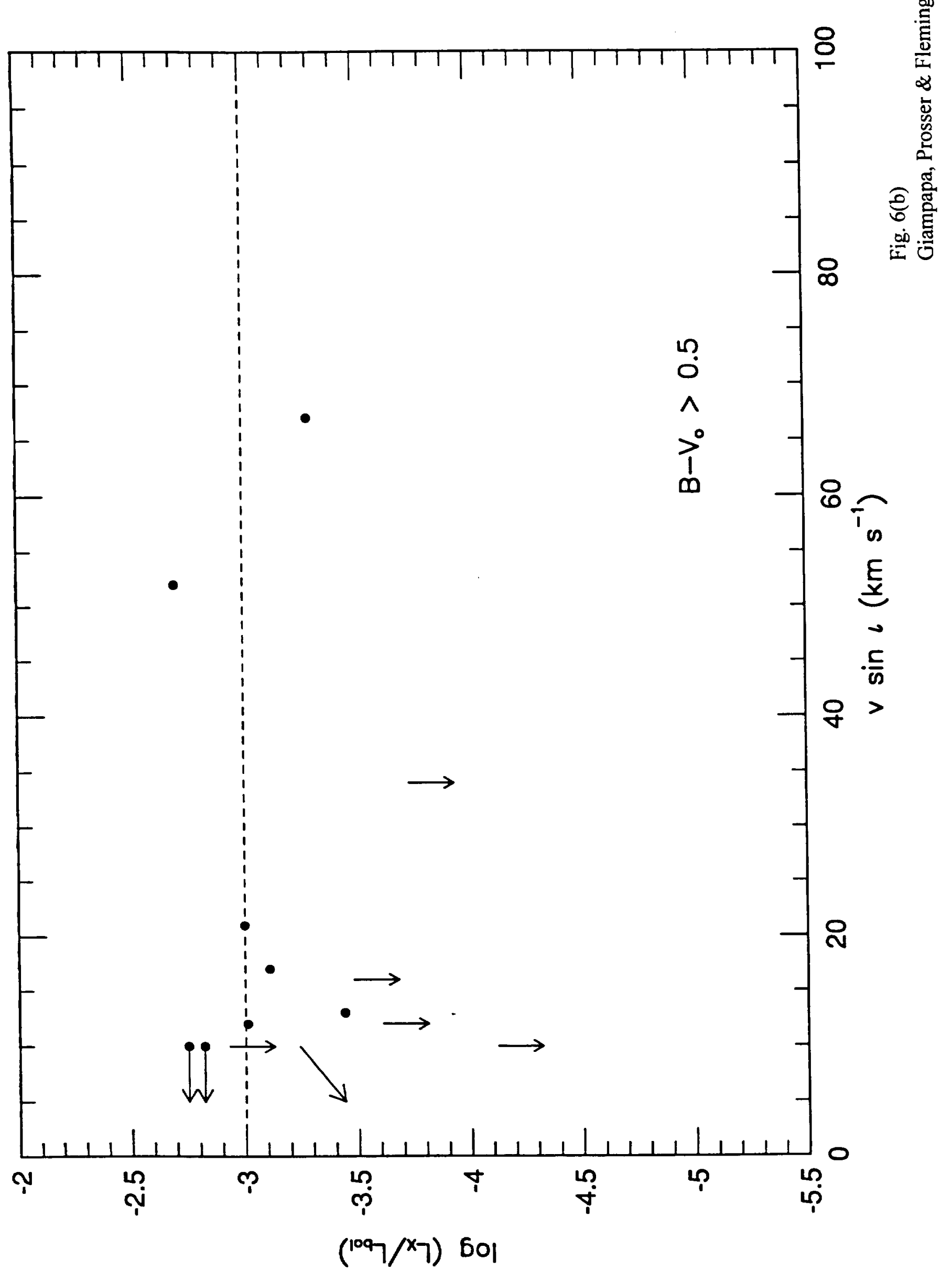




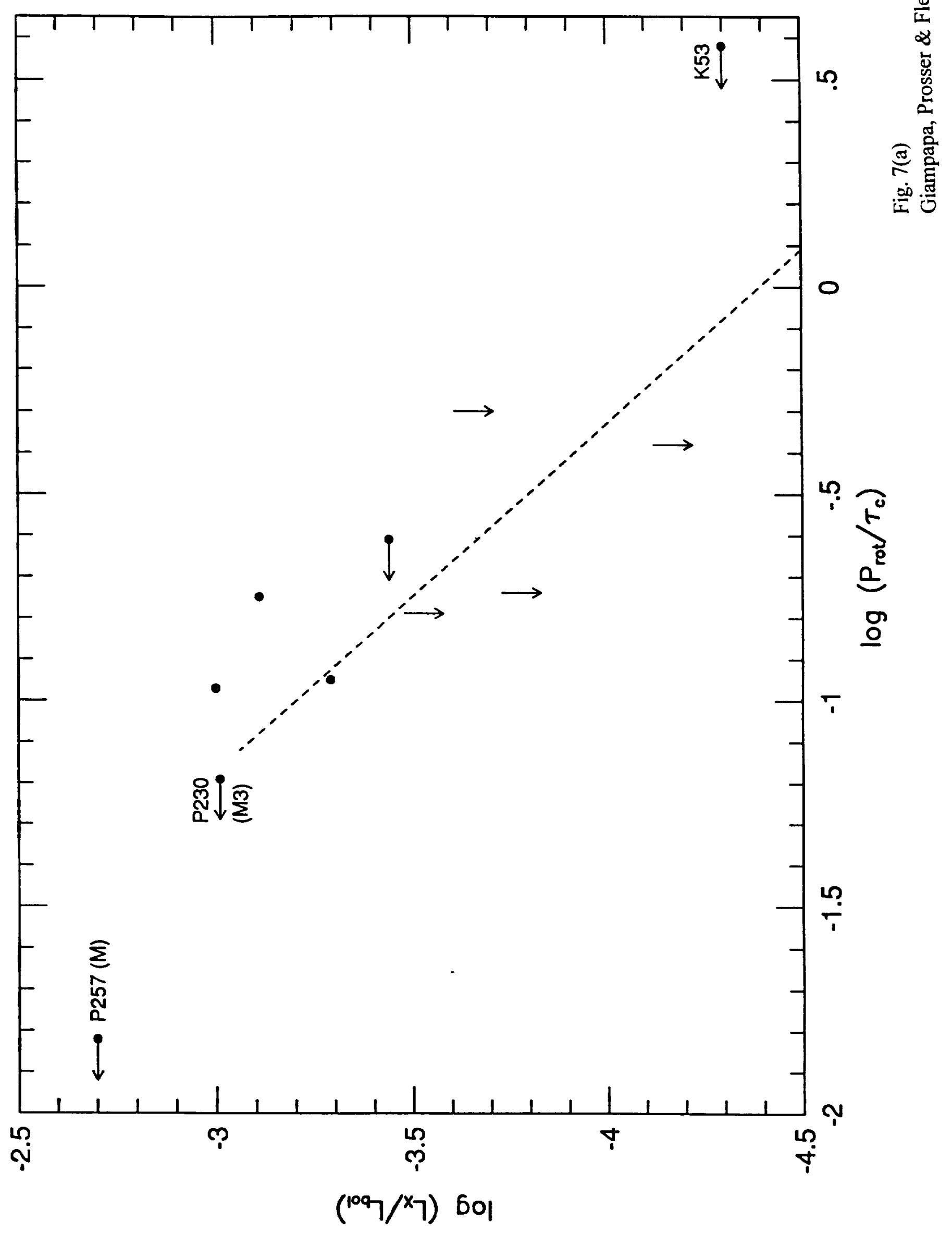




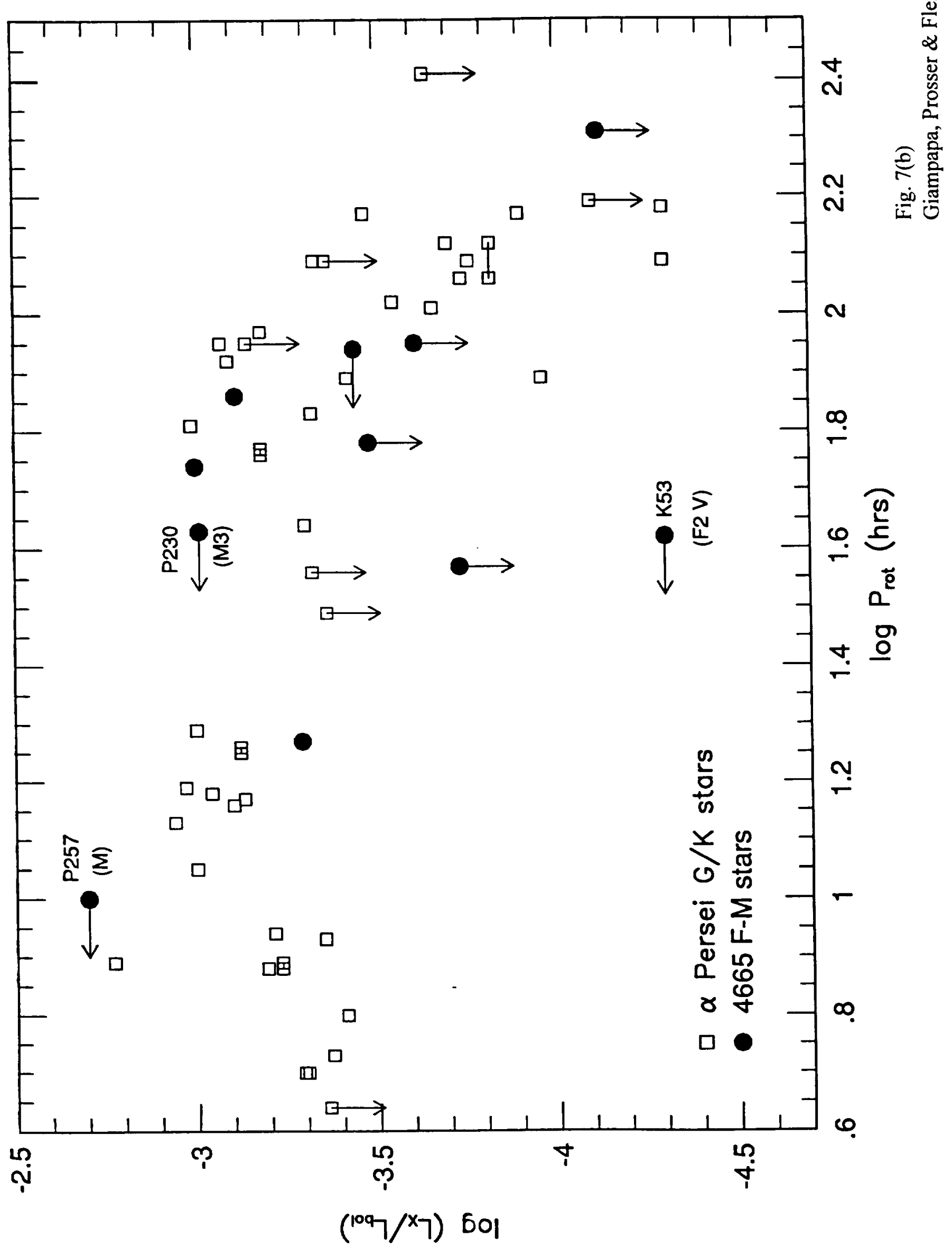




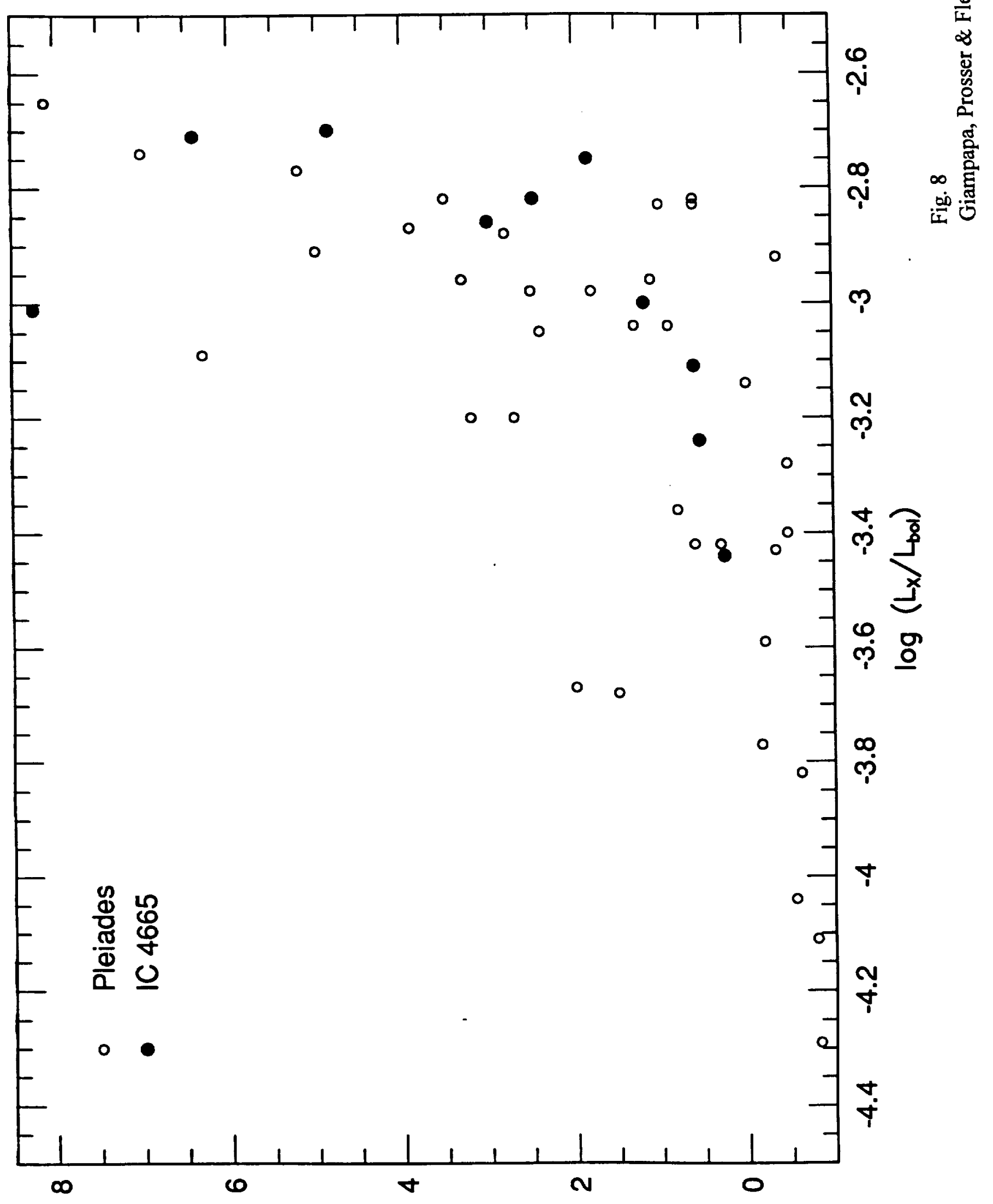

(y) ${ }^{{ }^{\circ} M}$ 


\begin{tabular}{|c|c|c|c|}
\hline 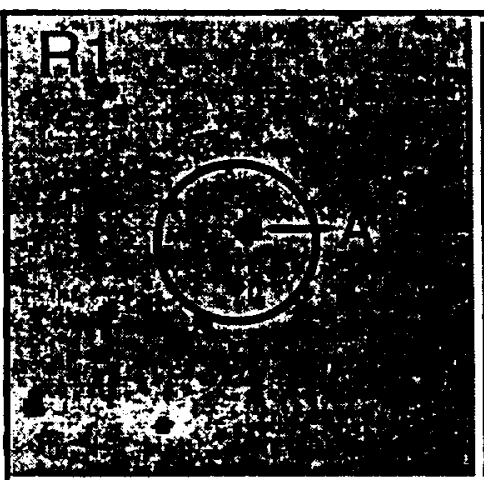 & 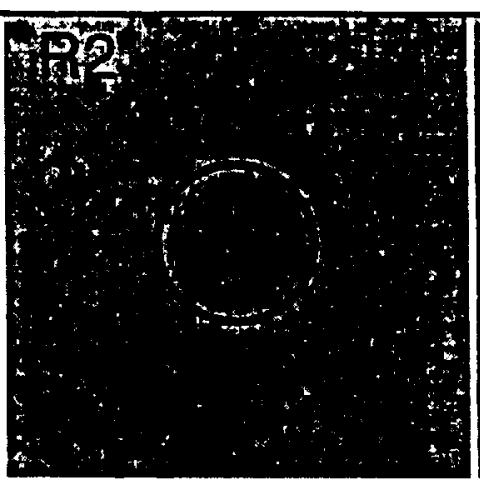 & a & 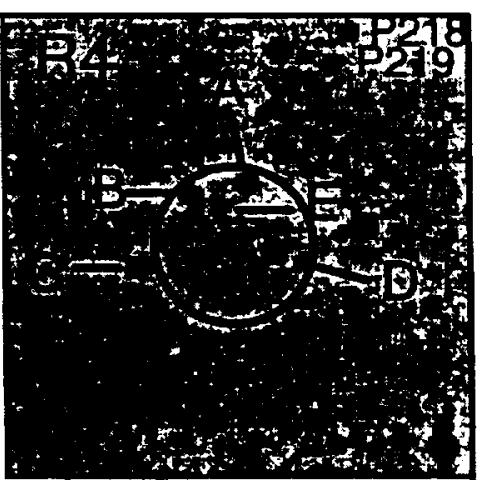 \\
\hline 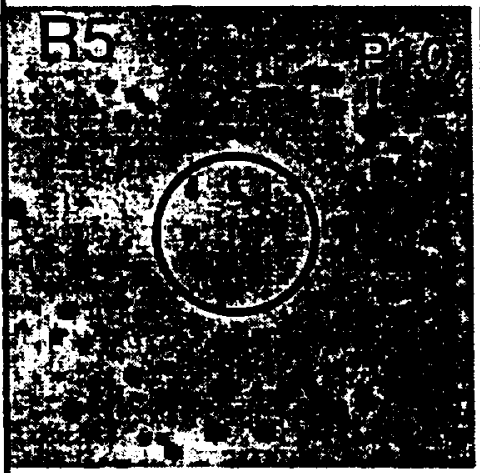 & 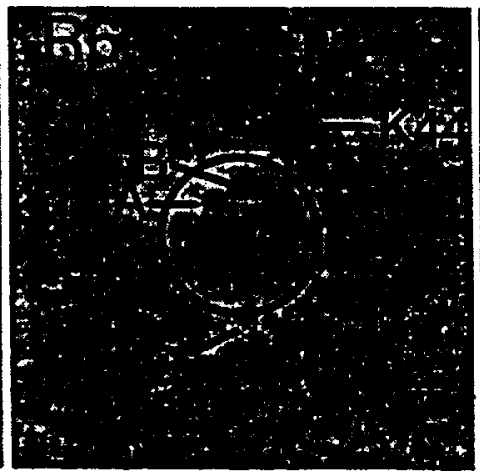 & 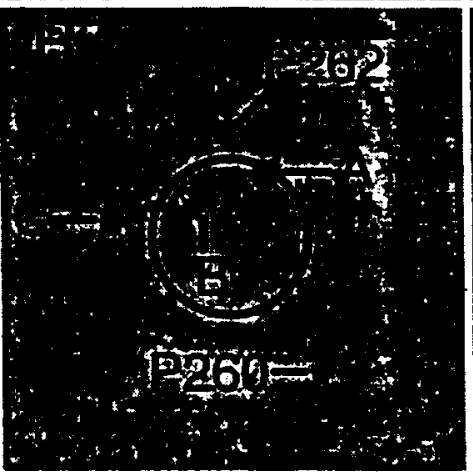 & 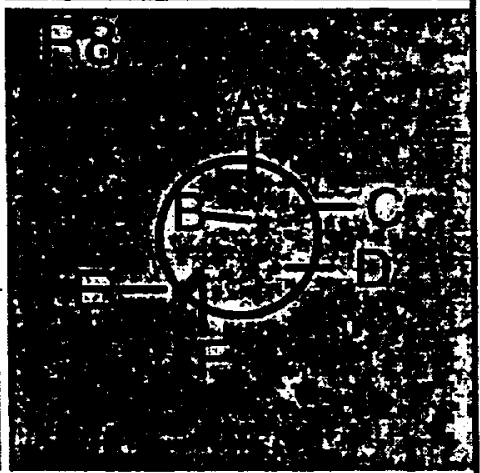 \\
\hline 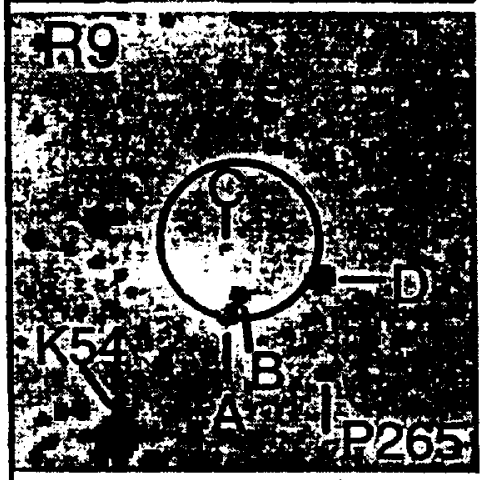 & 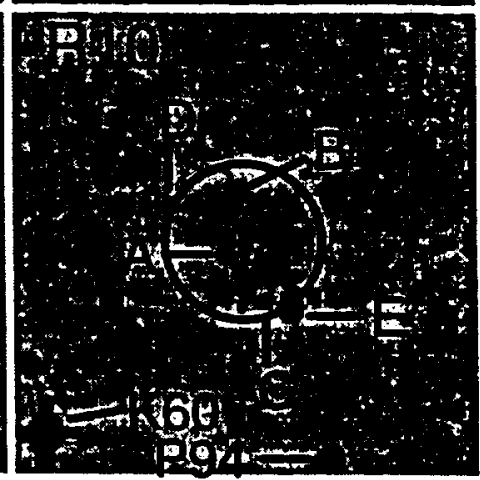 & ( & 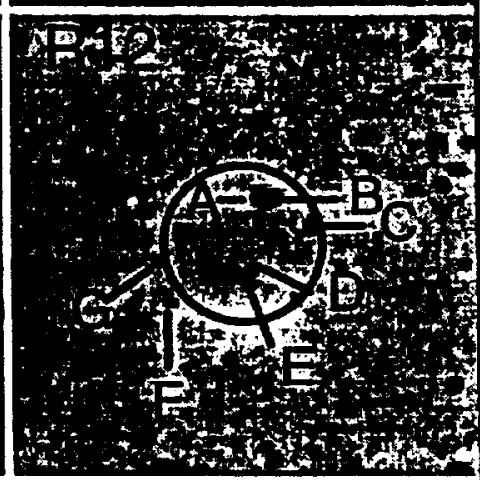 \\
\hline 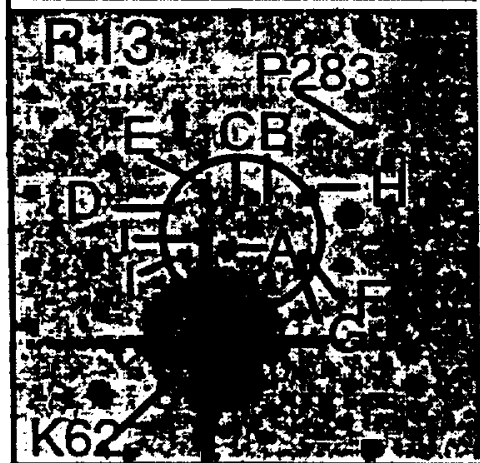 & 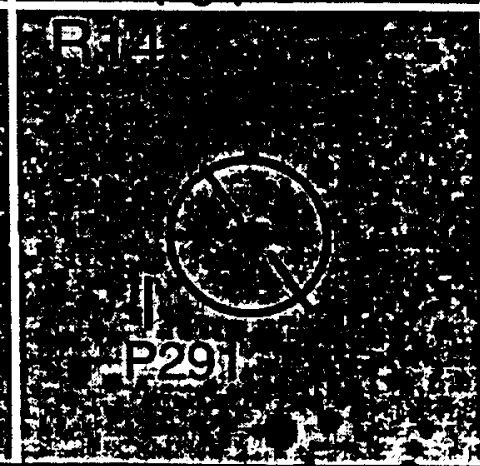 & (1) & \\
\hline
\end{tabular}

Fig. 9

Giampapa, Prosser \& Fleming (1997 
